\title{
Antivírus dotado de Rede Neural Artificial visando Detectar Malwares Preventivamente
}

\section{Title: Antivirus endowed with Artificial Neural Network in order to Detect Malwares Preventively}

\author{
Sidney M. L. Lima ', Heverton K. de L. Silva ${ }^{2}$, João H. da S. Luz ${ }^{2}$, Samuel L. de P. \\ Silva ${ }^{3}$, Hercília J. do N. Lima ${ }^{3}$, Anna B. A. de Andrade ${ }^{3}$, Alisson M. da Silva ${ }^{3}$ \\ 'Departamento de Computação, Universidade de Pernambuco - Recife, Brasil \\ Empresa Bidweb Security IT - Recife, Brasil \\ UniSãoMiguel, Curso de Segurança da Informação - Recife, Brasil \\ smll@ecomp.poli.br, \{hevertonkleidson155, joaohenriqu4, samuel1d2013, \\ herciliajuliana99, beatrizaugusta25, alisson_marek\}@gmail.com
}

\begin{abstract}
Malware is a software whose main goal is to access an device without the explicit permission of its owner. Antivirus is the most popular mechanism in order to detect malwares. Commercial antiviruses have as strategy to wait for a user to be infected and in sequence to report an anomalous behavior of her/his device, so that the antivirus manufacturer can take action. Then, in order to overcome the limitations of commercial antivirus, the proposed work creates an antivirus capable of preemptively identifying the modus operandi of a malicious application. The proposed antivirus employs artificial intelligence and can detect a malicious application even before it is run by the user. On average, the smart antivirus created can distinguish malware applications from benign ones in $98.13 \%$ of cases.
\end{abstract}

Keywords. Malwares; Antivirus; Artificial Neural Networks; Real-time malware detection; Computer Forensics.

Resumo. O malware é um software o qual tem como principal objetivo acessar um dispositivo alheio sem permissão explícita de seu proprietário. Os antivírus constituem o mecanismo mais popular quanto à detecção de malwares. Os antivírus comerciais têm como estratégia aguardar que algum usuário seja infectado e em sequência denuncie um comportamento anômalo de seu dispositivo, para que, então, a fabricante do antivírus possa tomar providências. Então, visando suprir as limitações dos antivírus comerciais, o trabalho proposto cria um antivírus capaz de identificar, preventivamente, o modus operandi de uma aplicação maliciosa. O antivírus proposto emprega inteligência artificial e consegue detectar um aplicativo mal-intencionado antes mesmo dele ser executado pelo usuário. Em média, o antivírus inteligente criado consegue distinguir os aplicativos malwares dos benignos em 98,13\% dos casos. 
Palavras-Chave. Malwares; Antivírus; Redes Neurais Artificiais; Detecção de Malwares em tempo real; Forense computacional.

\section{Introdução}

"Malware" é uma junção dos termos "malicioso" e "software". O malware tem como principal objetivo acessar um dispositivo alheio sem permissão explícita de seu proprietário. Segundo o Centro de Estudos, Respostas e Tratamento de Segurança (CERT.BR, 2016), de 2013 para 2014, o número de notificações de cyber-ataques reportadas à entidade aumentou $197 \%$. Aumentos semelhantes a esse tem se repetido em uma sequência de anos seguidos. Com a crescente popularização da internet, a tendência é de que esses números continuem crescendo de forma rápida, ainda durante alguns anos, visto que a internet é o principal meio de propagação de aplicações maliciosas (CERT.BR, 2016).

Sem dúvidas, os antivírus constituem o mecanismo mais popular quanto à segurança de informação. Eles estão presentes em $95 \%$ dos computadores pessoais e são associados ao combate de aplicações mal intencionadas (MICROSOFT, 2014). Tecnicamente, o vírus é apenas uma categoria dentre outras quanto a malwares como worm, backdoor, cavalo de troia, spyware, rootkits, ransomware, entre outros. Ao invés de antimalware, o termo antivírus costuma ser aplicado para definir ferramentas computacionais que visam prevenir, detectar e eliminar os malwares e seus malefícios.

O modus operandi dos antivírus comerciais é majoritariamente a identificação do executável malicioso com base em assinaturas (FILHO, et al., 2011). Isso quer dizer, o executável suspeito tem seu nome comparado a uma lista negra confeccionada a partir de denúncias prévias. Além disso, alguns antivírus comerciais dividem esse executável suspeito em pequenas porções (chunks). Assim, se um ou mais chunks do executável suspeito estiver presente na base de dados do antivírus, logo ele é classificado como malware (FILHO, et al., 2011). Cabe ressaltar que a comparação entre chunks da aplicação suspeita e a base de dados do antivírus pode se tornar inviável visto que são criados milhões de malwares anualmente (INTEL, 2017). Logo, averiguar a presença de partes de um executável suspeito em todos os milhões de exemplares maliciosos, possivelmente, é um problema computacional inviável a um usuário comum visto que essa tarefa poderia durar dias ou meses.

Outro grande problema dessa estratégia, adotada pelos antivírus comerciais, é para que haja a detecção de uma nova praga virtual é requerido que algumas máquinas já tenham sido infectadas. Cabe ressaltar que não basta apenas a detecção e eliminação do executável malicioso para que a vítima esteja livre de sua atuação. Além da eliminação do malware, é necessário desfazer todas as suas malfeitorias como, por exemplo, ter desabilitado os mecanismos de defesa da vítima, inclui-se firewall, plugins de segurança e os próprios antivírus. Logo, não é apropriada a estratégia de aguardar que uma vítima seja infectada e, em sequência denuncie um comportamento anômalo de seu dispositivo, para, então, tomar-se providências quanto à detecção de um novo malware. Enfatiza-se que uma única pessoa ou instituição infectada pode ter prejuízos irrecuperáveis.

Inclui-se como adversidade, no combate a aplicações mal intencionadas, o fato dos antivírus comerciais não possuírem um padrão na classificação dos malwares (FILHO, et al., 2011). Dessa forma, o tempo em que as fabricantes reagem a uma nova praga é afetado drasticamente. Como não existe um padrão, os antivírus dão os nomes 
que desejam, por exemplo, uma empresa pode categorizar um malware como "Malware.1" e uma segunda empresa classificá-lo como "Malware12310". Logo, a falta de um padrão, além do não compartilhamento de informações entre as fabricantes de antivírus, dificulta a detecção rápida e eficaz de uma aplicação mal intencionada.

Dadas as deficiências dos antivírus convencionais, o trabalho proposto investiga (i) 86 antivírus comerciais. A detecção de malwares variou entre $0 \%$ a 99,11\%, a depender do antivírus avaliado. Em média, houve a detecção $54,84 \%$ das pragas virtuais. Com aspecto desfavorável, os antivírus, em média, atestaram falsos negativos e foram omissos em $14,34 \%$ e $30,82 \%$ dos casos, respectivamente. Além disso, cerca de $17 \%$ dos antivírus não foram capazes de diagnosticar qualquer uma das amostras maliciosas. Enfatiza-se que os malwares analisados, no trabalho proposto, são de domínio público e têm as suas atuações maliciosas largamente documentadas pelos institutos de cyber-pesquisas (REWEMA, 2019). Mesmo assim, quase a quinta parte dos antivírus comerciais avaliados não tinham qualquer conhecimento sobre as existências dos malwares investigados. Notase, a limitação dos antivírus tradicionais quanto à robustez de serviços em tempo real e em larga escala.

De modo a suprir as limitações do antivírus comerciais, o estado-da-arte propõe extrair características do arquivo, de maneira preventiva, antes de executá-lo. $\mathrm{O}$ executável passa por um processo de disassembling visando reverter o arquivo binário em seu código fonte. Logo, o algoritmo, referente ao executável, pode ser estudado e, portanto, é possível investigar a intenção maliciosa do arquivo suspeito (ADKINS, et al., 2013), (ALAZAB, et al., 2015), (KHODAMORADI, et al., 2015), (FAN, et al., 2016). Então, a extração de características do algoritmo, referente ao arquivo periciado, possibilita a definição de uma sequencia de eventos suspeitos. Dessa forma, é possível determinar a intenção maliciosa do arquivo antes mesmo dele ser executado pelo usuário. Através da análise do algoritmo, o estado-da-arte investiga se o arquivo auditado cria, exclui, altera e faz o download de outros arquivos pela internet. Além disso, o estudo do algoritmo torna viável o rastreamento preventivo do tráfego de rede provocado pelo arquivo suspeito.

Após a extração das características dos executáveis suspeitos, o estado-da-arte costuma empregar modelos heurísticos onde são atribuídos pesos ponderados, de maneira empírica, às características dos arquivos auditados. A meta é agrupar os aplicativos suspeitos em duas classes: benigno e malwares. PRADO, et al. (2016), por exemplo, atribui pesos de 1 a 5 a comportamentos empiricamente classificados como suspeitos. $\mathrm{Na}$ obra de PRADO, et al. (2016), há a atribuição do peso 2, de forma heurística, caso o arquivo auditado tente criar um novo arquivo visto que esse comportamento pode estar atrelado a pragas virtuais. Modelos empíricos e heurísticos conseguem bons resultados mesmo sendo baseados na intuição do analista. Como efeito colateral, o empirismo pode provocar algumas distorções como, por exemplo, uma grande quantidade de falsos positivos. Ao empregar o empirismo, aproximadamente 1 em cada 5 executáveis benignos são equivocadamente classificados como malware (PRADO, et al., 2016).

As limitações de modelos heurísticos podem ser supridas por técnicas de inteligência artificial baseadas em aprendizado de máquina. Então, ao invés da intuição do analista, o peso ponderado referente a um comportamento suspeito é determinado através de máquinas de aprendizado estatístico. Inteligência artificial consegue automatizar de forma inteligente muitas tarefas, analisando milhares de arquivos, 
extraindo características deles e os ponderando estatisticamente. A inteligência artificial tem sido largamente aplicada nas mais diversas áreas, porém segurança da informação não é uma delas (HENKE, et al., 2011). Inteligência artificial pode contribuir bastante para o avanço da segurança em dispositivos. Há iniciativas, mas ainda se encontram em um estágio inicial (HENKE, et al., 2011).

Visando suprir as limitações e imprecisões dos modelos empíricos e heurísticos, o projeto proposto emprega redes neurais como técnica de inteligência artificial baseada em máquinas de aprendizado estatístico. Logo, o trabalho proposto tem como meta identificar, de forma estatística, comportamentos previamente classificados como suspeitos em um tempo de resposta viável a um usuário comum. Então, não é necessário aguardar meses ou anos até que o executável suspeito seja denunciado por um cliente assim como os antivírus comerciais atuam.

Quanto ao ambiente experimental, o trabalho proposto cria (ii) a REWEMA (Retrieval of 32-bit Windows Architecture Executables Applied to Malware Analysis Redistribuição de Executáveis do Windows em Arquiteturas de 32-bits Aplicados a Análise de Malware) (REWEMA, 2019). Por disputas comerciais, as fabricantes dos antivírus não compartilham, entre elas, as suas respectivas listas negras de malwares. A base de dados REWEMA é empregada como repositório do antivírus inteligente criado. A REWEMA apresenta 3136 executáveis malignos, visando arquiteturas de 32 bits, com suas respectivas análises emitidas pelo antivírus comerciais extraídas pela plataforma VirusTotal. A REWEMA disponibiliza outros 3136 executáveis benignos os quais, em conjunto com as amostragens malignas, são empregados no aprendizado através de redes neurais artificiais.

No tocante aos experimentos, os executáveis são divididos em duas classes: benigno e malwares. Na etapa de extração de características, são analisados 630 comportamentos e rotinas suspeitas extraídas do algoritmo pertencentes ao arquivo auditado. Na etapa de classificação, há o reconhecimento de padrão dos comportamentos atrelados a atividades maliciosas, especificamente, através de Redes Neurais Artificiais MLP (Multilayer Perceptron - Perceptron com Múltiplas Camadas) (LIMA, et al., 2016). As características extraídas dos executáveis servem como atributos de entrada das redes neurais artificiais no intuito de tornar o antivírus inteligente capaz de reconhecer atividades maliciosas antes mesmo de ser executado pelo usuário. $\mathrm{O}$ trabalho proposto e alcança um desempenho médio de $98,13 \%$ na distinção entre executáveis benignos e malwares, acompanhado de um tempo de resposta médio de apenas 0,06 segundos.

\section{Limitação dos Antivírus Comerciais}

Apesar de ser questionado há mais de uma década, o modus operandi dos antivírus é baseado em assinaturas quando o arquivo suspeito é consultado em bases de dados nomeadas de lista negra (SANS, 2018). Logo, basta que o hash do arquivo investigado não esteja na lista negra do antivírus para que o malware não seja detectado. O hash funciona como um identificador único de um dado arquivo. Então, dadas as limitações dos antivírus comerciais, não é uma tarefa difícil desenvolver e distribuir variantes de uma aplicação mal intencionada. Para isso, basta fazer pequenas alterações no malware original com rotinas que, efetivamente, não tem qualquer utilidade a exemplo de laços de repetição e desvios condicionais sem instruções em seus escopos. Essas alterações sem utilidade, no entanto, tornam o hash do malware modificado diferente do hash do 
malware original. Consequentemente, o malware, incrementado com rotinas nulas, não será detectado pelo antivírus o qual catalogou o malware original. Cabe ressaltar a existência de programas (exploits) responsáveis por criar e distribuir variantes, de forma automatizada, de um mesmo malware original. Conclui-se que antivírus, baseados em assinaturas, têm efetividade nula quando submetidos a variantes de um mesmo malware (SANS, 2018).

Por intermédio da plataforma VirusTotal', o trabalho proposto investiga 86 antivírus comerciais com seus respectivos resultados apresentados da Tabela 1 à Tabela 4. Foram empregados 3136 executáveis binários maliciosos obtidos da base de dados autoral nomeada REWEMA (REWEMA, 2019). O objetivo do trabalho é verificar a quantidade de pragas virtuais catalogadas pelos antivírus. A motivação é que a aquisição de novas pragas virtuais assume papel importante no combate a aplicações malintencionadas. Logo, quanto maior for a base de dados de malwares, nomeada de lista negra, melhor tende a ser a defesa provida pelo antivírus. A Figura 2 exibe o diagrama da metodologia proposta em diagrama de blocos. Inicialmente, os malwares são enviados ao servidor pertencente à plataforma VirusTotal. Após isso, os malwares são analisados pelos 86 antivírus comerciais vinculados ao VirusTotal. Logo, os antivírus provêm seus diagnósticos para os malwares submetidos à plataforma. Conforme ilustra a Figura 1, o VirusTotal permite a possibilidade de emissão de três tipos diferentes de diagnósticos; malware, benigno e omissão.

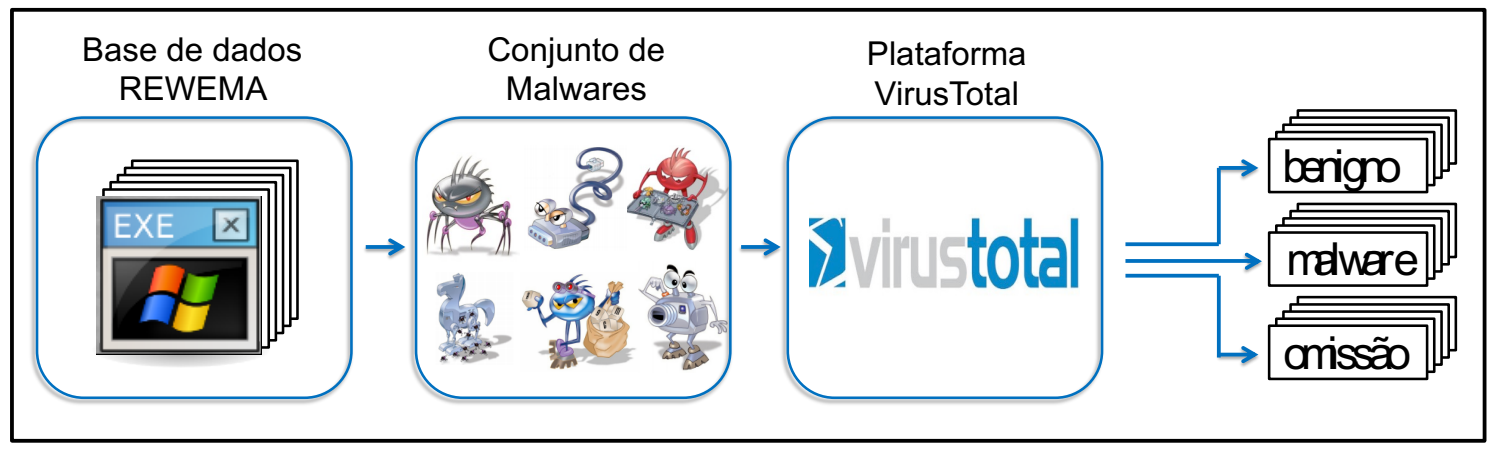

Figura 1. Diagrama da API VirusTotal.

Quanto à primeira possibilidade do VirusTotal, o antivírus detecta a malignidade do arquivo suspeito. No ambiente experimental proposto, todos os arquivos submetidos são malwares documentados por institutos de cyber-pesquisa (REWEMA, 2019). Logo, o antivírus acerta quando detecta a malignidade do arquivo investigado. A detecção do malware indica que o antivírus provê um serviço robusto contra cyber-invasões. Na segunda possibilidade, o antivírus atesta a benignidade do arquivo investigado. Logo, no estudo proposto, quando o antivírus alega a benignidade do arquivo, trata-se de um caso de falso negativo visto que todas as amostras são maliciosas. Isso quer dizer, o arquivo investigado é malware, no entanto, o antivírus atesta benignidade, de forma equivocada. $\mathrm{Na}$ terceira possibilidade, o antivírus não emite opinião sobre o arquivo suspeito. A omissão indica que o arquivo investigado jamais foi avaliado pelo antivírus tão pouco ele possui robustez para avaliá-lo em tempo real. A omissão do diagnóstico, por parte do antivírus, aponta a sua limitação quanto a serviços em larga escala.

\footnotetext{
VirusTotal: Serviço online quanto à identificação de malwares pelos principais antivírus comerciais mundiais. Disponível em: https://www.virustotal.com. Acesso em julho de 2018.
} 
Da Tabela 1 à Tabela 4, há a exibição dos resultados alcançados pelos 86 antivírus comerciais avaliados. O antivírus McAfee-GW-Edition obteve o melhor desempenho sendo capaz de detectar $99.11 \%$ dos malwares investigados. Uma grande adversidade, no combate a aplicações mal intencionadas, é o fato das fabricantes dos antivírus não compartilharem, entre elas, as suas respectivas listas negras de malwares devido a disputas comerciais. Através da análise da Tabela 1 à Tabela 4, o trabalho proposto aponta para um agravante dessa adversidade; uma mesma fabricante de antivírus sequer compartilha as suas bases de dados entre seus distintos antivírus. Observe, por exemplo, que os antivírus McAfee-GW-Edition e McAfee pertencem a uma mesma empresa. As suas listas negras, apesar de robustas, não são compartilhadas entre si. Logo, as estratégias comerciais, de uma mesma empresa, atrapalham o enfretamento a malwares. Complementa-se que as fabricantes de antivírus não estão necessariamente preocupadas em evitar as cyber-invasões, e sim em otimizar seus rendimentos comerciais.

A detecção de malwares variou entre $0 \%$ e $99,11 \%$, a depender do antivírus investigado. Em média, os 86 antivírus foram capazes de detectar 54,84\% das pragas virtuais avaliadas, com desvio padrão de 39,67. O desvio padrão elevado indica que a detecção de arquivos maliciosos pode sofrer variações abruptas a depender do antivírus escolhido. Determina-se que a proteção, contra invasões cibernéticas, está em função da escolha de um antivírus robusto dotado de uma grande e atualizada lista negra. Em média, os antivírus atestaram falsos negativos em 14,34\% dos casos, com desvio padrão de 21,67. Atestar a benignidade de um malware pode implicar em prejuízos irrecuperáveis. Uma pessoa ou instituição, por exemplo, passaria a confiar em uma determinada aplicação maliciosa quando, de fato, trata-se de um malware. Ainda como aspecto desfavorável, cerca de $17 \%$ dos antivírus não emitiram opinião em qualquer uma das 3136 amostras maliciosas. Em média, os antivírus foram omissos em 30,82\% dos casos, com desvio padrão de 40,97. A omissão do diagnóstico aponta a limitação dos antivírus quanto à detecção de malwares em tempo real.

Inclui-se como adversidade, no combate a aplicações mal intencionadas, o fato dos antivírus comerciais não possuírem um padrão na classificação dos malwares como visto da Tabela 5 à Tabela 10. Então, foram escolhidos 3 dos 3136 malwares de modo a exemplificar a miscelânea de classificações dadas pelos antivírus comerciais. Como não existe um padrão, os antivírus dão os nomes que desejam, por exemplo, uma empresa pode categorizar um malware como "Malware.1" e uma segunda empresa classificá-lo como "Malware12310". Logo, a falta de um padrão atrapalha as estratégias de cybervigilância visto que cada categoria de malware deveria ter tratamentos (vacinas) distintos. É importante ressaltar que uma mesma fabricante de antivírus pode não compartilhar, entre seus produtos, as suas bases de dados e também as suas categorias de malwares. Observe, por exemplo, que os antivírus McAfee-GW-Edition e McAfee pertencem a uma mesma empresa. Como efeito adverso, um mesmo malware pode ser classificado como "BehavesLike.Win32.Downloader.lc" e "W32/Sdbot.gen.an" pelo McAfee-GW-Edition e McAfee, respectivamente. Enfatiza-se que, no site oficial da fabricante McAfee, não há qualquer documentação sobre tais categorias de malwares. Conclui-se que, devido às estratégias comerciais, as fabricantes de antivírus atrapalham as estratégias de cyberproteção.

Ressalta-se que é inviável o aprendizado de máquina supervisionado visando reconhecimento de padrão de categorias de malwares. Devido a esse emaranhado confuso, visto da Tabela 5 à Tabela 10, é estatisticamente improvável que alguma técnica 
de aprendizado de máquina adquira capacidade de generalização. $\mathrm{O}$ aprendizado supervisionado deveria prover classificações de milhares de multi-classes (labels) providas pelos especialistas (antivírus).

Tabela 1. Parte 1: Resultado dos 86 antivírus comerciais.

\begin{tabular}{|c|c|c|c|}
\hline Antivírus & Detecção (\%) & Falso negativo (\%) & Omissão (\%) \\
\hline McAfee-GW-Edition & $99,11 \%$ & $0,89 \%$ & $0,00 \%$ \\
\hline McAfee & $98,98 \%$ & $1,02 \%$ & $0,00 \%$ \\
\hline Panda & $98,76 \%$ & $1,15 \%$ & $0,10 \%$ \\
\hline Comodo & $98,37 \%$ & $1,59 \%$ & $0,03 \%$ \\
\hline Kaspersky & $98,34 \%$ & $1,56 \%$ & $0,10 \%$ \\
\hline NANO-Antivirus & $98,25 \%$ & $1,69 \%$ & $0,06 \%$ \\
\hline Symantec & $97,64 \%$ & $2,26 \%$ & $0,10 \%$ \\
\hline GData & $97,23 \%$ & $2,77 \%$ & $0,51 \%$ \\
\hline BitDefender & $97,23 \%$ & $2,77 \%$ & $0,00 \%$ \\
\hline MicroWorld-eScan & $96,94 \%$ & $2,74 \%$ & $0,32 \%$ \\
\hline F-Secure & $98,76 \%$ & $3,00 \%$ & $1,12 \%$ \\
\hline Qihoo-360 & $95,66 \%$ & $1,69 \%$ & $2,65 \%$ \\
\hline VIPRE & $95,54 \%$ & $4,46 \%$ & $0,00 \%$ \\
\hline Sophos & $95,38 \%$ & $4,53 \%$ & $0,10 \%$ \\
\hline Emsisoft & $95,22 \%$ & $4,21 \%$ & $0,57 \%$ \\
\hline Avira & $95,18 \%$ & $2,39 \%$ & $2,42 \%$ \\
\hline $\mathrm{CMC}$ & $95,15 \%$ & $4,37 \%$ & $0,48 \%$ \\
\hline Arcabit & $94,58 \%$ & $4,21 \%$ & $1,21 \%$ \\
\hline DrWeb & $94,36 \%$ & $5,61 \%$ & $0,03 \%$ \\
\hline Ad-Aware & $93,88 \%$ & $3,32 \%$ & $2,81 \%$ \\
\hline Fortinet & $93,85 \%$ & $6,15 \%$ & $0,00 \%$ \\
\hline Rising & $93,72 \%$ & $5,01 \%$ & $1,28 \%$ \\
\hline Antiy-AVL & $92,38 \%$ & $7,21 \%$ & $0,41 \%$ \\
\hline Jiangmin & $91,87 \%$ & $8,04 \%$ & $0,10 \%$ \\
\hline Ikarus & $90,50 \%$ & $8,20 \%$ & $1,31 \%$ \\
\hline
\end{tabular}

Tabela 2. Parte 2: Resultado dos 86 antivírus comerciais. 


\begin{tabular}{|c|c|c|c|}
\hline Antivírus & Detecção (\%) & Falso negativo (\%) & Omissão (\%) \\
\hline Cyren & $90,34 \%$ & $8,67 \%$ & $0,99 \%$ \\
\hline Zillya & $89,89 \%$ & $8,32 \%$ & $1,79 \%$ \\
\hline F-Prot & $89,51 \%$ & $10,49 \%$ & $0,00 \%$ \\
\hline TheHacker & $87,69 \%$ & $12,28 \%$ & $0,03 \%$ \\
\hline Avast & $86,19 \%$ & $13,71 \%$ & $0,10 \%$ \\
\hline ESET-NOD32 & $86,13 \%$ & $13,87 \%$ & $0,00 \%$ \\
\hline AVG & $85,55 \%$ & $14,45 \%$ & $0,00 \%$ \\
\hline Microsoft & $85,20 \%$ & $14,80 \%$ & $0,00 \%$ \\
\hline AegisLab & $82,40 \%$ & $17,12 \%$ & $0,48 \%$ \\
\hline Tencent & $82,21 \%$ & $6,44 \%$ & $11,35 \%$ \\
\hline VBA32 & $81,12 \%$ & $18,88 \%$ & $0,00 \%$ \\
\hline TrendMicro-HouseCal & $79,02 \%$ & $17,83 \%$ & $3,16 \%$ \\
\hline K7AntiVirus & $78,95 \%$ & $21,05 \%$ & $0,00 \%$ \\
\hline K7GW & $78,83 \%$ & $21,17 \%$ & $0,00 \%$ \\
\hline AVware & $78,64 \%$ & $4,02 \%$ & $17,25 \%$ \\
\hline TrendMicro & $78,54 \%$ & $18,53 \%$ & $2,93 \%$ \\
\hline ALYac & $77,20 \%$ & $16,55 \%$ & $6,25 \%$ \\
\hline Yandex & $70,22 \%$ & $3,19 \%$ & $26,59 \%$ \\
\hline AhnLab-V3 & $69,29 \%$ & $30,52 \%$ & $0,19 \%$ \\
\hline nProtect & $67,22 \%$ & $32,49 \%$ & $0,29 \%$ \\
\hline ViRobot & $64,06 \%$ & $35,94 \%$ & $0,00 \%$ \\
\hline ClamAV & $59,41 \%$ & $40,31 \%$ & $0,29 \%$ \\
\hline ZoneAlarm & $54,37 \%$ & $1,02 \%$ & $44,61 \%$ \\
\hline Webroot & $52,04 \%$ & $2,20 \%$ & $45,76 \%$ \\
\hline MAX & $50,77 \%$ & $1,66 \%$ & $47,58 \%$ \\
\hline Cylance & $48,60 \%$ & $2,87 \%$ & $48,53 \%$ \\
\hline TotalDefense & $41,04 \%$ & $53,13 \%$ & $5,84 \%$ \\
\hline CrowdStrike & $40,88 \%$ & $21,78 \%$ & $37,34 \%$ \\
\hline
\end{tabular}

Tabela 3. Parte 3: Resultado dos 86 antivírus comerciais. 


\begin{tabular}{|c|c|c|c|}
\hline Antivírus & Detecção (\%) & Falso negativo (\%) & Omissão (\%) \\
\hline Invincea & $40,27 \%$ & $27,04 \%$ & $32,68 \%$ \\
\hline Endgame & $40,18 \%$ & $14,99 \%$ & $44,83 \%$ \\
\hline Baidu & $39,48 \%$ & $34,98 \%$ & $25,54 \%$ \\
\hline CAT-QuickHeal & $39,19 \%$ & $60,81 \%$ & $0,00 \%$ \\
\hline Agnitum & $25,22 \%$ & $0,80 \%$ & $73,98 \%$ \\
\hline Bkav & $22,39 \%$ & $73,02 \%$ & $4,59 \%$ \\
\hline SentinelOne & $20,92 \%$ & $32,49 \%$ & $46,59 \%$ \\
\hline Kingsoft & $19,87 \%$ & $57,59 \%$ & $22,54 \%$ \\
\hline Paloalto & $17,60 \%$ & $37,12 \%$ & $45,28 \%$ \\
\hline SUPERAntiSpyware & $7,33 \%$ & $92,67 \%$ & $0,00 \%$ \\
\hline Malwarebytes & $7,21 \%$ & $92,19 \%$ & $0,61 \%$ \\
\hline 1ByteHero & $2,36 \%$ & $23,50 \%$ & $74,14 \%$ \\
\hline Zoner & $2,17 \%$ & $96,65 \%$ & $1,18 \%$ \\
\hline Norman & $1,18 \%$ & $0,03 \%$ & $98,79 \%$ \\
\hline AntiVir & $0,99 \%$ & $0,00 \%$ & $99,01 \%$ \\
\hline Commtouch & $0,89 \%$ & $0,10 \%$ & $99,01 \%$ \\
\hline Ahnlab & $0,03 \%$ & $0,00 \%$ & $99,97 \%$ \\
\hline Alibaba & $0,00 \%$ & $35,49 \%$ & $64,51 \%$ \\
\hline VirusBuster & $0,00 \%$ & $0,00 \%$ & $100,00 \%$ \\
\hline NOD32 & $0,00 \%$ & $0,00 \%$ & $100,00 \%$ \\
\hline eSafe & $0,00 \%$ & $0,00 \%$ & $100,00 \%$ \\
\hline eTrust-Vet & $0,00 \%$ & $0,00 \%$ & $100,00 \%$ \\
\hline Authentium & $0,00 \%$ & $0,00 \%$ & $100,00 \%$ \\
\hline Prevx & $0,00 \%$ & $0,00 \%$ & $100,00 \%$ \\
\hline Sunbelt & $0,00 \%$ & $0,00 \%$ & $100,00 \%$ \\
\hline PCTools & $0,00 \%$ & $0,00 \%$ & $100,00 \%$ \\
\hline Squared & $0,00 \%$ & $0,00 \%$ & $100,00 \%$ \\
\hline WhiteArmor & $0,00 \%$ & $0,00 \%$ & $100,00 \%$ \\
\hline
\end{tabular}

Tabela 4. Parte 4: Resultado dos 86 antivírus comerciais. 


\begin{tabular}{l|l|l|l}
\hline Antivírus & Detecção (\%) & Falso negativo (\%) & Omissão (\%) \\
\hline Command & $0,00 \%$ & $0,00 \%$ & $100,00 \%$ \\
\hline SAVMail & $0,00 \%$ & $0,00 \%$ & $100,00 \%$ \\
\hline FileAdvisor & $0,00 \%$ & $0,00 \%$ & $100,00 \%$ \\
\hline Ewido & $0,00 \%$ & $0,00 \%$ & $100,00 \%$ \\
\hline Webwasher-Gateway & $0,00 \%$ & $0,00 \%$ & $100,00 \%$ \\
\hline
\end{tabular}

Tabela 5. Parte 1: Miscelânea de classificações providas pelos antivírus comerciais.

\begin{tabular}{|c|c|c|c|}
\hline Antivírus & Backdoor.IRC.exe & $\begin{array}{l}\text { AndroRat } \\
\text { Binder_Patched.exe }\end{array}$ & Rustock.C.exe \\
\hline $\begin{array}{l}\text { McAfee-GW- } \\
\text { Edition }\end{array}$ & $\begin{array}{l}\text { BehavesLike.Win32.Do } \\
\text { wnloader.lc }\end{array}$ & RDN/Generic.grp & $\begin{array}{l}\text { BehavesLike.Win32.Ramn } \\
\text { it.kc }\end{array}$ \\
\hline McAfee & W32/Sdbot.gen.an & RDN/Generic.grp & $\begin{array}{l}\text { Generic.dx!FDAFB3A143 } \\
38\end{array}$ \\
\hline Panda & Trj/Genetic.gen & Trj/GdSda.A & Trj/Agent.EDT \\
\hline Comodo & Backdoor.IRC.Bot-gen & UnclassifiedMalware & $\begin{array}{l}\text { Trojan- } \\
\text { Clicker.Win32.Costrat.bk }\end{array}$ \\
\hline Kaspersky & $\begin{array}{l}\text { Backdoor.Win32.IRCB } \\
\text { ot.gen }\end{array}$ & $\begin{array}{l}\text { UDS:DangerousObject.Mu } \\
\text { lti.Generic }\end{array}$ & $\begin{array}{l}\text { Trojan- } \\
\text { Clicker.Win32.Costrat.bk }\end{array}$ \\
\hline $\begin{array}{l}\text { NANO- } \\
\text { Antivirus }\end{array}$ & $\begin{array}{l}\text { Trojan.Win32.IRCBot.d } \\
\text { kptuu }\end{array}$ & $\begin{array}{l}\text { Riskware.Win32.Androrat. } \\
\text { ebhzgr }\end{array}$ & $\begin{array}{l}\text { Trojan.Win32.Spambot.eat } \\
\text { xnv }\end{array}$ \\
\hline Symantec & W32.IRCBot & Trojan.Gen.2 & Backdoor.Rustock.B \\
\hline GData & $\begin{array}{l}\text { Generic.Malware.SIBdl } \\
\text { dg.28B98B92 }\end{array}$ & $\begin{array}{l}\text { Trojan.GenericKD.340770 } \\
8\end{array}$ & Backdoor.Rustock.Gen.1 \\
\hline BitDefender & $\begin{array}{l}\text { Generic.Malware.SIBdl } \\
\text { dg.28B98B92 }\end{array}$ & $\begin{array}{l}\text { Trojan.GenericKD.340770 } \\
8\end{array}$ & Backdoor.Rustock.Gen.1 \\
\hline $\begin{array}{l}\text { MicroWorld- } \\
\text { eScan }\end{array}$ & $\begin{array}{l}\text { Generic.Malware.SIBdl } \\
\text { dg.28B98B92 }\end{array}$ & $\begin{array}{l}\text { Trojan.GenericKD.340770 } \\
8\end{array}$ & Backdoor.Rustock.Gen.1 \\
\hline F-Secure & $\begin{array}{l}\text { Generic.Malware.SIBdl } \\
\text { dg.28B98B92 }\end{array}$ & $\begin{array}{l}\text { Trojan.GenericKD.340770 } \\
8\end{array}$ & Backdoor.Rustock.Gen.1 \\
\hline Qihoo-360 & Omission & Win32/Trojan.125 & Win32/Trojan.Clicker.89e \\
\hline VIPRE & $\begin{array}{l}\text { Trojan.Win32.Ircbot!co } \\
\text { bra (v) }\end{array}$ & Trojan.Win32.Generic!BT & Trojan.Win32.Generic!BT \\
\hline Sophos & W32/Sdbot-Gen & Generic PUA BL (PUA) & Mal/RKRustok-A \\
\hline Emsisoft & $\begin{array}{l}\text { Generic.Malware.SIBdl } \\
\text { dg.28B98B92 (B) }\end{array}$ & $\begin{array}{l}\text { Trojan.GenericKD. } 340770 \\
8 \text { (B) }\end{array}$ & $\begin{array}{l}\text { Backdoor.Rustock.Gen.1 } \\
\text { (B) }\end{array}$ \\
\hline Avira & WORM/SdBot.13824 & TR/NetSeal.575488 & TR/Dropper.Gen \\
\hline
\end{tabular}

Tabela 6. Parte 2: Miscelânea de classificações providas pelos antivírus comerciais. 


\begin{tabular}{|c|c|c|c|}
\hline Antivírus & Backdoor.IRC.exe & $\begin{array}{l}\text { AndroRat } \\
\text { Binder_Patched.exe }\end{array}$ & Rustock.C.exe \\
\hline $\mathrm{CMC}$ & $\begin{array}{l}\text { Generic.Win32.fee } 5446 \\
\text { b04!MD }\end{array}$ & Benign & $\begin{array}{l}\text { Generic.Win } 32.56 \mathrm{c} 37100 \mathrm{c} \\
\mathrm{e} ! \mathrm{MD}\end{array}$ \\
\hline Arcabit & $\begin{array}{l}\text { Generic.Malware.SIBdl } \\
\text { dg.28B98B92 }\end{array}$ & Trojan.Generic.D33FF5C', & Backdoor.Rustock.Gen.1 \\
\hline DrWeb & Win32.IRC.Bot.based & Tool.Androrat & Trojan.Spambot \\
\hline Ad-Aware & $\begin{array}{l}\text { Generic.Malware.SIBdl } \\
\text { dg.28B98B92 }\end{array}$ & $\begin{array}{l}\text { Trojan.GenericKD.340770 } \\
8\end{array}$ & Backdoor.Rustock.Gen.1 \\
\hline Fortinet & W32/SDBot.ABC!tr.bdr & $\begin{array}{l}\text { MSIL/Generic.DN.1135A0 } \\
\text { !tr }\end{array}$ & W32/Generic.CON!tr \\
\hline Rising & $\begin{array}{l}\text { PE:Malware.Generic(Th } \\
\text { under)!1.A1C4 }[\mathrm{F}]\end{array}$ & $\begin{array}{l}\text { Trojan.Generic } \\
\text { (cloud:rtYiZ8N6s3M) }\end{array}$ & $\begin{array}{l}\text { Backdoor.Rustock.i } \\
\text { (classic) }\end{array}$ \\
\hline Antiy-AVL & $\begin{array}{l}\text { Trojan[Backdoor]/IRC. } \\
\text { Bot-gen }\end{array}$ & Benign & Trojan/Win32.SGeneric \\
\hline Jiangmin & Backdoor/IRC.Bot-gen & Benign & TrojanClicker.Costrat.lx \\
\hline Ikarus & Backdoor.Win32.SdBot & PUA.MSIL.NetSeal & Backdoor.WinNT.Rustock \\
\hline Cyren & $\begin{array}{l}\text { W32/Bloop.A.gen!Eldo } \\
\text { rado }\end{array}$ & Benign & W32/Rustock.CQKI-9104 \\
\hline Zillya & Backdoor.Bot.Win32.15 & $\begin{array}{l}\text { Adware.MegaSearch.Win3 } \\
2.17554\end{array}$ & Trojan.Castrat.Win32.62 \\
\hline F-Prot & $\begin{array}{l}\text { W32/Bloop.A.gen!Eldo } \\
\text { rado }\end{array}$ & Benign & W32/Rustock.C' \\
\hline TheHacker & IRC/SdBot & Benign & Trojan/Clicker.Costrat.bk \\
\hline Avast & $\begin{array}{l}\text { Win32:SdBot-gen17 } \\
\text { [Trj] }\end{array}$ & Win32:Malware-gen & Win32:Susn-F [Trj] \\
\hline ESET-NOD32 & IRC.Bot-gen & $\begin{array}{l}\text { A variant of } \\
\text { MSIL/Packed.NetSeal.A } \\
\text { suspicious }\end{array}$ & $\begin{array}{l}\text { a variant of } \\
\text { Win32/Rootkit.Kryptik.BP }\end{array}$ \\
\hline AVG & $\begin{array}{l}\text { IRC/BackDoor.SdBot. } \\
\text { MP }\end{array}$ & Win32:Malware-gen & Crypt5.ANAA \\
\hline Microsoft & Backdoor:Win32/Sdbot & $\begin{array}{l}\text { MonitoringTool:AndroidO } \\
\text { S/AndroRat }\end{array}$ & $\begin{array}{l}\text { Backdoor:WinNT/Rustock. } \\
\text { D }\end{array}$ \\
\hline AegisLab & Benign & Gen.Variant.Barys!c & $\begin{array}{l}\text { Troj.Clicker.W32.Costrat.b } \\
\text { k!c }\end{array}$ \\
\hline Tencent & $\begin{array}{l}\text { Win32.Backdoor.Ircbot. } \\
\text { Eivd }\end{array}$ & Benign & $\begin{array}{l}\text { Win32.Trojan.Costrat.Egy } \\
\mathrm{h}\end{array}$ \\
\hline VBA32 & $\begin{array}{l}\text { BScope.Backdoor.Win } 3 \\
2 . \text { SdBot }\end{array}$ & Benign & $\begin{array}{l}\text { Malware- } \\
\text { Cryptor.Win32.015 }\end{array}$ \\
\hline $\begin{array}{l}\text { TrendMicro- } \\
\text { HouseCal }\end{array}$ & BKDR_SDBOT.A & $\begin{array}{l}\text { TROJ_GEN.ROC1COEDK } \\
17\end{array}$ & BKDR_RUSTOCK.AR \\
\hline
\end{tabular}

Tabela 7. Parte 3: Miscelânea de classificações providas pelos antivírus comerciais. 


\begin{tabular}{|c|c|c|c|}
\hline Antivírus & Backdoor.IRC.exe & $\begin{array}{l}\text { AndroRat } \\
\text { Binder_Patched.exe }\end{array}$ & Rustock.C.exe \\
\hline Tencent & $\begin{array}{l}\text { Win32.Backdoor.Ircbot. } \\
\text { Eivd }\end{array}$ & Benign & $\begin{array}{l}\text { Win32.Trojan.Costrat.Egy } \\
\text { h }\end{array}$ \\
\hline VBA32 & $\begin{array}{l}\text { BScope.Backdoor.Win3 } \\
\text { 2.SdBot }\end{array}$ & Benign & $\begin{array}{l}\text { Malware- } \\
\text { Cryptor.Win32.015 }\end{array}$ \\
\hline $\begin{array}{l}\text { TrendMicro- } \\
\text { HouseCal }\end{array}$ & BKDR_SDBOT.A & $\begin{array}{l}\text { TROJ_GEN.R0C1C0EDK } \\
17\end{array}$ & BKDR_RUSTOCK.AR \\
\hline $\begin{array}{l}\text { McAfee-GW- } \\
\text { Edition }\end{array}$ & $\begin{array}{l}\text { BehavesLike.Win32.Do } \\
\text { wnloader.lc }\end{array}$ & RDN/Generic.grp & $\begin{array}{l}\text { BehavesLike.Win32.Ramn } \\
\text { it.kc }\end{array}$ \\
\hline McAfee & W32/Sdbot.gen.an & RDN/Generic.grp & $\begin{array}{l}\text { Generic.dx!FDAFB3A143 } \\
38\end{array}$ \\
\hline Panda & Trj/Genetic.gen & Trj/GdSda.A & Trj/Agent.EDT \\
\hline Comodo & Backdoor.IRC.Bot-gen & UnclassifiedMalware & $\begin{array}{l}\text { Trojan- } \\
\text { Clicker.Win32.Costrat.bk }\end{array}$ \\
\hline Kaspersky & $\begin{array}{l}\text { Backdoor.Win32.IRCB } \\
\text { ot.gen }\end{array}$ & $\begin{array}{l}\text { UDS:DangerousObject.Mu } \\
\text { lti.Generic }\end{array}$ & $\begin{array}{l}\text { Trojan- } \\
\text { Clicker.Win32.Costrat.bk }\end{array}$ \\
\hline $\begin{array}{l}\text { NANO- } \\
\text { Antivirus }\end{array}$ & $\begin{array}{l}\text { Trojan.Win32.IRCBot.d } \\
\text { kptuu }\end{array}$ & $\begin{array}{l}\text { Riskware.Win32.Androrat. } \\
\text { ebhzgr }\end{array}$ & $\begin{array}{l}\text { Trojan.Win32.Spambot.eat } \\
\text { xnv }\end{array}$ \\
\hline Symantec & W32.IRCBot & Trojan.Gen.2 & Backdoor.Rustock.B \\
\hline GData & $\begin{array}{l}\text { Generic.Malware.SIBdl } \\
\text { dg.28B98B92 }\end{array}$ & $\begin{array}{l}\text { Trojan.GenericKD.340770 } \\
8\end{array}$ & Backdoor.Rustock.Gen.1 \\
\hline BitDefender & $\begin{array}{l}\text { Generic.Malware.SIBdl } \\
\text { dg.28B98B92 }\end{array}$ & $\begin{array}{l}\text { Trojan.GenericKD.340770 } \\
8\end{array}$ & Backdoor.Rustock.Gen.1 \\
\hline $\begin{array}{l}\text { MicroWorld- } \\
\text { eScan }\end{array}$ & $\begin{array}{l}\text { Generic.Malware.SIBdl } \\
\text { dg.28B98B92 }\end{array}$ & $\begin{array}{l}\text { Trojan.GenericKD. } 340770 \\
8\end{array}$ & Backdoor.Rustock.Gen.1 \\
\hline F-Secure & $\begin{array}{l}\text { Generic.Malware.SIBdl } \\
\text { dg.28B98B92 }\end{array}$ & $\begin{array}{l}\text { Trojan.GenericKD.340770 } \\
8\end{array}$ & Backdoor.Rustock.Gen.1 \\
\hline Qihoo-360 & Omission & Win32/Trojan.125 & Win32/Trojan.Clicker.89e \\
\hline VIPRE & $\begin{array}{l}\text { Trojan.Win32.Ircbot!co } \\
\text { bra (v) }\end{array}$ & Trojan.Win32.Generic!BT & Trojan.Win32.Generic!BT \\
\hline Sophos & W32/Sdbot-Gen & Generic PUA BL (PUA) & Mal/RKRustok-A \\
\hline Emsisoft & $\begin{array}{l}\text { Generic.Malware.SIBdl } \\
\text { dg.28B98B92 (B) }\end{array}$ & $\begin{array}{l}\text { Trojan.GenericKD. } 340770 \\
8 \text { (B) }\end{array}$ & $\begin{array}{l}\text { Backdoor.Rustock.Gen.1 } \\
\text { (B) }\end{array}$ \\
\hline Avira & WORM/SdBot.13824 & TR/NetSeal.575488 & TR/Dropper.Gen \\
\hline CMC & $\begin{array}{l}\text { Generic.Win32.fee } 5446 \\
\text { b04!MD }\end{array}$ & Benign & $\begin{array}{l}\text { Generic.Win } 32.56 \mathrm{c} 37100 \mathrm{c} \\
\text { e!MD }\end{array}$ \\
\hline Arcabit & $\begin{array}{l}\text { Generic.Malware.SIBdl } \\
\text { dg.28B98B92 }\end{array}$ & Trojan.Generic.D33FF5C', & Backdoor.Rustock.Gen.1 \\
\hline DrWeb & Win32.IRC.Bot.based & Tool.Androrat & Trojan.Spambot \\
\hline
\end{tabular}

Tabela 8. Parte 4: Miscelânea de classificações providas pelos antivírus comerciais. 


\begin{tabular}{|c|c|c|c|}
\hline Antivírus & Backdoor.IRC.exe & $\begin{array}{l}\text { AndroRat } \\
\text { Binder_Patched.exe }\end{array}$ & Rustock.C.exe \\
\hline Ad-Aware & $\begin{array}{l}\text { Generic.Malware.SIBdl } \\
\text { dg.28B98B92 }\end{array}$ & $\begin{array}{l}\text { Trojan.GenericKD. } 340770 \\
8\end{array}$ & Backdoor.Rustock.Gen.1 \\
\hline Fortinet & W32/SDBot.ABC!tr.bdr & $\begin{array}{l}\text { MSIL/Generic.DN.1135A0 } \\
\text { !tr }\end{array}$ & W32/Generic.CON!tr \\
\hline Rising & $\begin{array}{l}\text { PE:Malware.Generic(Th } \\
\text { under)!1.A1C4 }[\mathrm{F}]\end{array}$ & $\begin{array}{l}\text { Trojan.Generic } \\
\text { (cloud:rtYiZ8N6s3M) }\end{array}$ & $\begin{array}{l}\text { Backdoor.Rustock.i } \\
\text { (classic) }\end{array}$ \\
\hline Antiy-AVL & $\begin{array}{l}\text { Trojan[Backdoor]/IRC. } \\
\text { Bot-gen }\end{array}$ & Benign & Trojan/Win32.SGeneric \\
\hline Jiangmin & Backdoor/IRC.Bot-gen & Benign & TrojanClicker.Costrat.lx \\
\hline Ikarus & Backdoor.Win32.SdBot & PUA.MSIL.NetSeal & Backdoor.WinNT.Rustock \\
\hline Cyren & $\begin{array}{l}\text { W32/Bloop.A.gen!Eldo } \\
\text { rado }\end{array}$ & Benign & W32/Rustock.CQKI-9104 \\
\hline Zillya & Backdoor.Bot.Win32.15 & $\begin{array}{l}\text { Adware.MegaSearch.Win3 } \\
2.17554\end{array}$ & Trojan.Castrat.Win32.62 \\
\hline F-Prot & $\begin{array}{l}\text { W32/Bloop.A.gen!Eldo } \\
\text { rado }\end{array}$ & Benign & W32/Rustock.C' \\
\hline TheHacker & IRC/SdBot & Benign & Trojan/Clicker.Costrat.bk \\
\hline Avast & $\begin{array}{l}\text { Win32:SdBot-gen } 17 \\
\text { [Trj] }\end{array}$ & Win32:Malware-gen & Win32:Susn-F [Trj] \\
\hline ESET-NOD32 & IRC.Bot-gen & $\begin{array}{l}\text { A variant of } \\
\text { MSIL/Packed.NetSeal.A } \\
\text { suspicious }\end{array}$ & $\begin{array}{l}\text { a variant of } \\
\text { Win32/Rootkit.Kryptik.BP }\end{array}$ \\
\hline AVG & $\begin{array}{l}\text { IRC/BackDoor.SdBot. } \\
\text { MP }\end{array}$ & Win32:Malware-gen & Crypt5.ANAA \\
\hline Microsoft & Backdoor:Win32/Sdbot & $\begin{array}{l}\text { MonitoringTool:AndroidO } \\
\text { S/AndroRat }\end{array}$ & $\begin{array}{l}\text { Backdoor:WinNT/Rustock. } \\
\text { D }\end{array}$ \\
\hline AegisLab & Benign & Gen.Variant.Barys!c & $\begin{array}{l}\text { Troj.Clicker.W32.Costrat.b } \\
\text { k!c }\end{array}$ \\
\hline Tencent & $\begin{array}{l}\text { Win32.Backdoor.Ircbot. } \\
\text { Eivd }\end{array}$ & Benign & $\begin{array}{l}\text { Win32.Trojan.Costrat.Egy } \\
\mathrm{h}\end{array}$ \\
\hline VBA32 & $\begin{array}{l}\text { BScope.Backdoor.Win3 } \\
\text { 2.SdBot }\end{array}$ & Benign & $\begin{array}{l}\text { Malware- } \\
\text { Cryptor.Win32.015 }\end{array}$ \\
\hline $\begin{array}{l}\text { TrendMicro- } \\
\text { HouseCal }\end{array}$ & BKDR_SDBOT.A & $\begin{array}{l}\text { TROJ_GEN.R0C1C0EDK } \\
17\end{array}$ & BKDR_RUSTOCK.AR \\
\hline K7AntiVirus & Backdoor ( 04c514e61) & Trojan ( 004b4bad1 ) & RootKit ( 004d16801) \\
\hline K7GW & Backdoor ( 04c514e61) & Trojan ( 004b4bad1 ) & RootKit ( 004d16801) \\
\hline AVware & Omission & Trojan.Win32.Generic!BT & Trojan.Win32.Generic!BT \\
\hline
\end{tabular}

Tabela 9. Parte 5: Miscelânea de classificações providas pelos antivírus comerciais. 


\begin{tabular}{|c|c|c|c|}
\hline Antivírus & Backdoor.IRC.exe & $\begin{array}{l}\text { AndroRat } \\
\text { Binder_Patched.exe }\end{array}$ & Rustock.C.exe \\
\hline TrendMicro & BKDR_SDBOT.A & $\begin{array}{l}\text { TROJ_GEN.R0C1C0EDK } \\
17\end{array}$ & Benign \\
\hline ALYac & Omission & $\begin{array}{l}\text { Trojan.GenericKD.340770 } \\
8\end{array}$ & Backdoor.Rustock.Gen.1 \\
\hline Yandex & Omission & Riskware.NetSeal! & $\begin{array}{l}\text { Trojan.Rustock!gMcRHMf } \\
\text { Fn+E }\end{array}$ \\
\hline AhnLab-V3 & $\begin{array}{l}\text { Win32/IRCBot.worm.v } \\
\text { ariant }\end{array}$ & Benign & Benign \\
\hline nProtect & $\begin{array}{l}\text { Backdoor/W32.Agent.1 } \\
\text { 3824.AL }\end{array}$ & Benign & $\begin{array}{l}\text { Trojan- } \\
\text { Clicker/W32.Costrat.70570 }\end{array}$ \\
\hline ViRobot & $\begin{array}{l}\text { Backdoor.Win32.Bot- } \\
\text { gen.13824[h] }\end{array}$ & $\begin{array}{l}\text { Trojan.Win32.Z.Netseal.57 } \\
5488[\mathrm{~h}]\end{array}$ & $\begin{array}{l}\text { Trojan.Win32.Clicker.705 } \\
\text { 70[h] }\end{array}$ \\
\hline ClamAV & Trojan.IRCBot.13856 & Benign & Win.Trojan.Clicker-1225 \\
\hline ZoneAlarm & Omission & $\begin{array}{l}\text { UDS:DangerousObject.Mu } \\
\text { lti.Generic }\end{array}$ & Omission \\
\hline Webroot & Omission & W32.Malware.Ml.Vt & W32.Rustock.Rootkit \\
\hline MAX & Omission & Omission & Omission \\
\hline Cylance & Omission & Omission & Omission \\
\hline TotalDefense & Win32/Sdbot!generic & Benign & Benign \\
\hline CrowdStrike & Omission & $\begin{array}{l}\text { malicious_confidence_74 } \\
\% \text { (D) }\end{array}$ & $\begin{array}{l}\text { malicious_confidence_100 } \\
\% \text { (D) }\end{array}$ \\
\hline Invincea & Omission & Benign & backdoor.winnt.rustock.d \\
\hline Endgame & Omission & Benign & $\begin{array}{l}\text { malicious (high } \\
\text { confidence) }\end{array}$ \\
\hline Baidu & $\begin{array}{l}\text { Backdoor.Win32.IRCB } \\
\text { ot.gen }\end{array}$ & Benign & $\begin{array}{l}\text { Win32.Trojan.WisdomEye } \\
\text { s.16070401.9500.9995 }\end{array}$ \\
\hline CAT-QuickHeal & Omission & Monitoringtool.Androrat & Backdoor.Rustock \\
\hline Agnitum & Backdoor.SdBot.gen & Omission & Omission \\
\hline Bkav & Benign & Benign & W32.Clodf61.Trojan.3118 \\
\hline SentinelOne & Omission & static engine - malicious & Omission \\
\hline Kingsoft & Omission & Benign & Benign \\
\hline Paloalto & Omission & generic.ml & Omission \\
\hline $\begin{array}{l}\text { SUPERAntiSpy } \\
\text { ware }\end{array}$ & Benign & Benign & Benign \\
\hline Malwarebytes & Benign & Benign & Benign \\
\hline ByteHero & Virus.Win32.Heur.c & Omission & Omission \\
\hline
\end{tabular}

Tabela 10. Parte 6: Miscelânea de classificações providas pelos antivírus comerciais. 


\begin{tabular}{|c|c|c|c|}
\hline Antivírus & Backdoor.IRC.exe & $\begin{array}{l}\text { AndroRat } \\
\text { Binder_Patched.exe }\end{array}$ & Rustock.C.exe \\
\hline Zoner & Benign & Benign & Benign \\
\hline Norman & Omission & Omission & Omission \\
\hline AntiVir & Backdoor ( 04c514e61) & Omission & RootKit ( 004d16801) \\
\hline Commtouch & Omission & Omission & Omission \\
\hline Ahnlab & $\begin{array}{l}\text { Win32/IRCBot.worm.v } \\
\text { ariant }\end{array}$ & Benign & Benign \\
\hline Alibaba & Benign & Omission & Omission \\
\hline VirusBuster & Omission & Omission & Omission \\
\hline NOD32 & Omission & $\begin{array}{l}\text { a variant of } \\
\text { MSIL/Packed.NetSeal.A } \\
\text { suspicious }\end{array}$ & $\begin{array}{l}\text { a variant of } \\
\text { Win32/Rootkit.Kryptik.BP }\end{array}$ \\
\hline eSafe & Omission & Omission & Omission \\
\hline eTrust-Vet & Omission & Omission & Omission \\
\hline Authentium & Omission & Omission & Omission \\
\hline Prevx & Omission & Omission & Omission \\
\hline Sunbelt & Omission & Omission & Omission \\
\hline PCTools & Omission & Omission & Omission \\
\hline Squared & Omission & Omission & Omission \\
\hline WhiteArmor & Omission & Omission & Omission \\
\hline Command & Omission & Omission & Omission \\
\hline SAVMail & Omission & Omission & Omission \\
\hline FileAdvisor & Omission & Omission & Omission \\
\hline Ewido & Omission & Omission & Omission \\
\hline $\begin{array}{l}\text { Webwasher- } \\
\text { Gateway }\end{array}$ & Omission & Omission & Omission \\
\hline
\end{tabular}

\section{Metodologia Proposta}

Dada as limitações dos antivírus comerciais, o trabalho proposto visa criar um antivírus, dotado de inteligência artificial, capaz de diferenciar aplicativos malwares de benignos de forma preventiva. A Figura 2 exibe o diagrama da metodologia proposta em diagrama de blocos.

Inicialmente, são extraídas as características do conjunto de executáveis. Logo, as redes neurais artificiais utilizam, como atributos de entrada, essas características extraídas das executáveis os quais são classificados entre benignos e malwares. Os resultados da classificação estão descritos no Capítulo 4. 


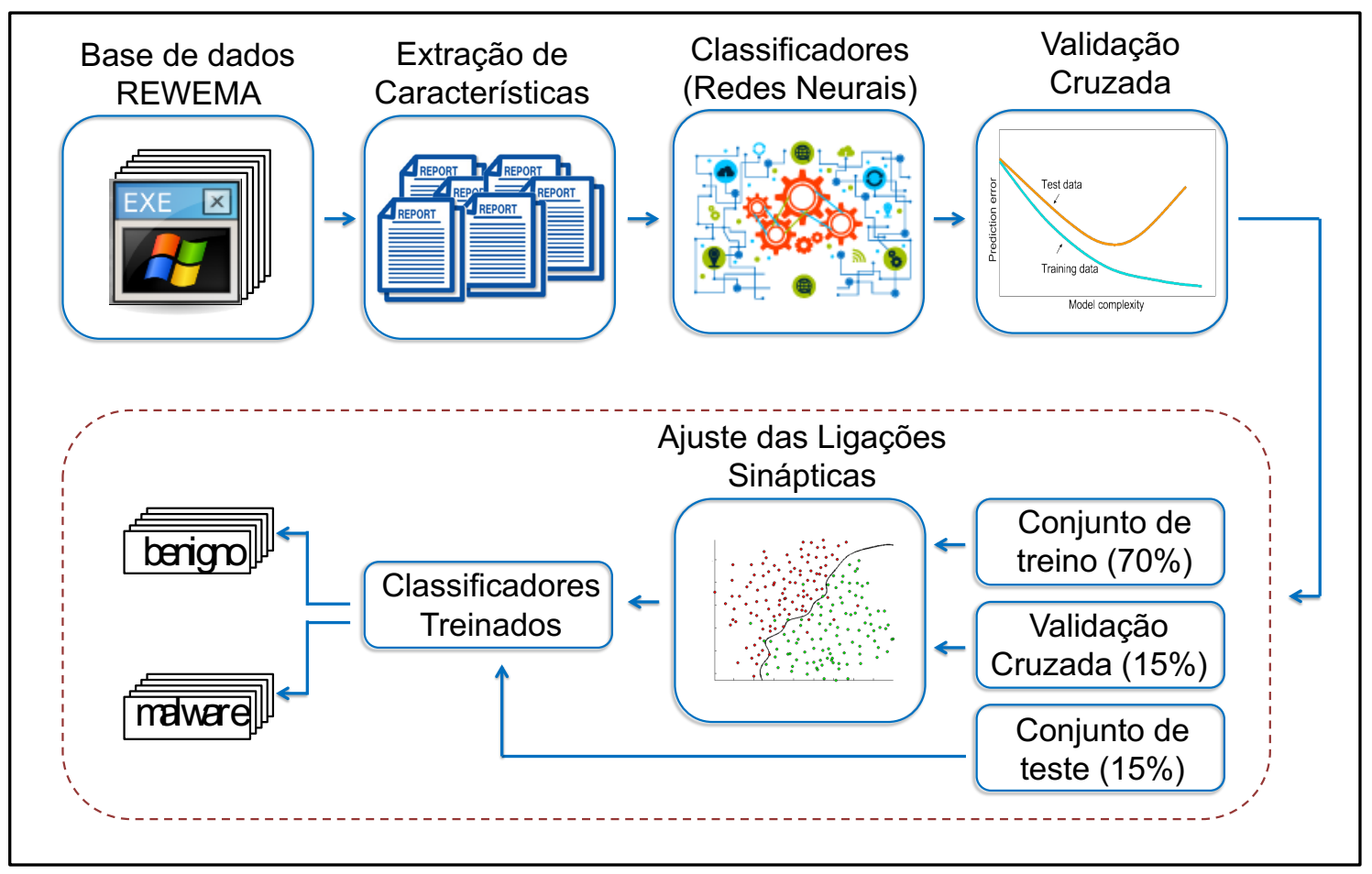

Figura 2. Diagrama da metodologia proposta.

\subsection{Materiais e Métodos: Base de Dados REWEMA}

O trabalho proposto cria uma base de dados de modo que o antivírus inteligente possa adquirir capacidade de aprendizado mediante as instancias apresentadas. A base de dados criada é batizada de REWEMA (Retrieval of 32-bit Windows Architecture Executables Applied to Malware Analysis - Redistribuição de Executáveis do Windows em Arquiteturas de 32-bits Aplicados a Análise de Malware) (REWEMA, 2019). Há 3316 executáveis malignos e outros 3316 executáveis benignos. Logo, a base REWEMA se torna adequada ao aprendizado dotado de inteligência artificial visto que ambas as classes de executáveis apresentam a mesma quantidade.

Quanto aos executáveis maliciosos, o REWEMA é a junção de várias bases de dados de malwares. As pragas virtuais foram extraídas de bases de dados disponibilizadas por grupos entusiastas do estudo de malwares, como o Vxheaven ${ }^{2} \mathrm{e}$ TheZoo ${ }^{3}$. Quanto aos executáveis benignos, a aquisição foi oriunda de repositórios de aplicações benignas como Sourceforge ${ }^{4}$, Github ${ }^{5}$ e Sysinternals ${ }^{6}$. Cabe ressaltar que todos os executáveis benignos foram submetidos ao VirusTotal e todos tiveram a sua benignidade atestada pelos principais antivírus comerciais mundiais. Os diagnósticos, providos pelos

\footnotetext{
2 Vxheaven: bases de dados quanto a executáveis malwares em arquiteturas de 32 bits. Disponível em: http://vxheaven.org/. Acesso em junho de 2017.

"TheZoo: bases de dados quanto a executáveis malwares em arquiteturas de 32 bits. Disponível em: https://github.com/ytisf/theZoo. Acesso em junho de 2018.

- Sourceforge: Repositório de aplicativos benignos. Disponível em: https://sourceforge.net/. Acesso em junho de 2018.

"Github: Repositório de aplicativos benignos Disponível em: https://github.com/. Acesso em junho de 2018. - Sysinternals: Repositório de aplicativos benignos. Disponível em: https://live.sysinternals.com/. Acesso em junho de 2018.
} 
VirusTotal, correspondentes aos executáveis benignos e malwares estão disponibilizados no endereço virtual da base de dados REWEMA (REWEMA, 2019).

O objetivo da criação da base de dados REWEMA é dar total possibilidade da metodologia proposta ser replicada, por terceiros, em trabalhos futuros. Logo, o artigo proposto, ao disponibilizar, livremente, a sua base de dados viabiliza transparência e imparcialidade à pesquisa, além de demostrar a veracidade dos resultados alcançados. Então, espera-se que a metodologia, a ser relatada no capítulo seguinte, sirva de base para a criação de novos trabalhos científicos.

\subsection{Extração de Características dos Executáveis}

A extração de características dos executáveis passa pelo processo de disassembling visando reverter o arquivo binário em seu código fonte. Logo, o algoritmo, referente ao executável, pode ser estudado e posteriormente classificado pelas redes neurais descritas na próxima seção. Da Tabela 11 à Tabela 13, há as descrições das características às suas respectivas ferramentas, funções e bibliotecas. No total, são extraídas 630 características, de cada executável, referentes aos grupos acima citados. Scripts autorais e a ferramenta pescanner ${ }^{\mathrm{F}}$ são utilizadas na extração de características dos executáveis. A seguir, são detalhados os grupos de características referentes ao algoritmo dos arquivos investigados.

Histograma das instruções responsáveis pela aquisição de dados (imports) ${ }^{\mathrm{F} 2}$.

Quantidade de sub-rotinas que invocam o TLS (Transport Layer Security).

$\checkmark$ Quantidade de sub-rotinas responsáveis pela exportação de dados (exports).

$\checkmark$ Histograma das importações das API (Application Programming Interface - Interface de Programação de Aplicações) empregadas pelo executável.

$\checkmark$ Características relacionadas a indícios que o computador tenha sofrido fragmentação no seu disco rígido, além de tentativas de inicialização inválidas acumuladas ${ }^{\text {в3. }}$.

$\checkmark$ Modo de execução do aplicativo. Há duas opções:

- softwares dotados de interface gráfica (GUI);

- softwares executados no console.

$\checkmark$ Características relacionadas ao Sistema Operacional. A forense digital averigua se o arquivo testado tenta:

- identificar o nome do usuário atual do sistema operacional ${ }^{\mathrm{k}}$;

- acessar funções de interface de programação de aplicativo (API) para criar e gerenciar perfis de usuário atual do $\mathrm{SO}$ F;

- detectar o número de milissegundos decorridos desde que o sistema foi inicializado ${ }^{\mathrm{F}}$;

- executar uma operação em um arquivo especifico ${ }^{\mathrm{F}}$;

- identificar a versão do Sistema Operacional Windows em uso ${ }^{\text {r8}}$;

- monitorar o tráfego de mensagens internas entre os processos do Sistema ${ }^{\text {rg}}$;

- alterar as configurações e conteúdos da inicialização (STARTUPINFO) do SO Windows ${ }^{\mathrm{Fi}}$;

- permitir que aplicativos acessem a funcionalidade fornecida pelo shell do sistema operacional, além de alterá-lo ${ }^{\mathrm{\mu l}}$;

- alterar as mensagens de logon na inicialização do SO Windows ${ }^{\mathrm{F} 2}$; 
- alterar aplicativos nativos atrelados a caixas de diálogo padrão para abrir e salvar arquivos, escolhendo cor e fonte, dentre outras customizações ${ }^{\mathrm{F} 3}$;

- configurar o licenciamento do Servidor Windows ${ }^{\mathrm{Fia}}$;

- configurar o Windows Server 2003 Fis;

- alterar as configurações de energia do Sistema ${ }^{\mathrm{F} 16}$;

- abrir um processo, serviço ou biblioteca nativa do Sistema Operacional ${ }^{\mathrm{Fr}}$;

- excluir o contexto de certificados vinculados ao Sistema Operacional ${ }^{\mathrm{Fs}}$;

- copiar um arquivo existente para um novo arquivo ${ }^{\mathrm{Fl}}$;

- criar, abrir, excluir ou alterar um arquivo ${ }^{\mathrm{F} 20}$;

- criar e executar novo(s) processo(s) ${ }^{\mathrm{F} 21}$.

- criar novo(s) diretório(s) ${ }^{\mathrm{F} 22}$;

- procurar por arquivo(s) específico(s) ${ }^{\mathrm{F} z}$;

- criar um objeto de serviço e o adicionar ao banco de dados do gerenciador de controle de um determinado serviço ${ }^{\mathrm{F} 24}$;

- criptografar dados. Tal estratégia é típica de ransomwares os quais sequestram os dados da vítima através da criptografia. Para descriptografar os dados, o invasor pede ao usuário um montante monetário para que possa ter de volta todos os seus dados ${ }^{\mathrm{r} 25}$

- acessar sistemas de arquivos, dispositivos, processos, threads e tratamento de erros ${ }^{\mathrm{r2} 6}$;

- alterar as propriedades de som e dispositivo de áudio do sistema ${ }^{\mathrm{r} 2}$;

- acessar informações de conteúdo gráfico para monitores, impressoras e outros dispositivos de saída do SO Windows ${ }^{\mathrm{rex}}$;

- usar e/ou monitorar a porta USB ${ }^{\mathrm{r} 29}$;

- controlar um driver de um determinado dispositivo ${ }^{\mathrm{F} 0}$;

- investigar se uma unidade de disco é uma unidade removível, fixa, de CD/DVDROM, de RAM ou de rede ${ }^{\text {rs }}$;

Características relacionadas ao Registro (Regedit) do SO Windows. Cabe ressaltar que a vítima pode não estar livre da infecção de um malware mesmo após a sua detecção e eliminação. A persistência das malfeitorias, mesmo após a exclusão do malware, ocorre devido à inserção de entradas (chaves) maliciosas no Regedit. Logo, quando o sistema operacional é inicializado, o cyber-ataque recomeça devido à chave mal-intencionada invocar a vulnerabilidade explorada pelo malware (eg: redirecionar a página inicial do Internet Explorer). Logo, o antivírus criado audita se o aplicativo suspeito tenta:

- detectar o nome NetBIOS do computador local. Esse nome é estabelecido na inicialização do sistema, quando o sistema o lê no registro (Regedit) ${ }^{\mathrm{F} 2}$;

- encerrar uma chave de um registro específico ${ }^{\mathrm{F} 3}$;

- criar uma chave de um registro específico. Caso a chave já exista no Regedit, então, ela será lida ${ }^{{ }^{34} \text {; }}$

- excluir uma chave e seus valores no Regedit ${ }^{\text {rss; }}$;

- enumerar e abrir as sub-chaves da chave de um registro aberto específico ${ }^{\mathrm{3} 36}$.

$\checkmark$ Características relacionadas a spywares como keyloggers (captura de informações do teclado visando o furto de senhas e logins) e screenloggers (filmagem da tela da vítima). $\mathrm{O}$ antivírus proposta audita se o arquivo analisado tenta: 
- detectar, em qual parte da tela da vítima, houver uma atualização ${ }^{\mathrm{F} 37}$;

- identificar a região de atualização da tela copiando-a para uma determinada região ${ }^{\mathrm{B} 3}$;

- capturar filmes e vídeos AVI de câmeras da Web e outros hardwares de vídeo ${ }^{\text {r39}}$;

- capturar informações quanto à votação eletrônica, especificamente, da empresa

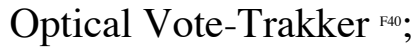

- copiar uma matriz de estados das teclas do teclado ${ }^{\mathrm{F} 4}$. Tal estratégia é típica dos keyloggers;

- monitorar a atividade da Internet do usuário e informações particulares ${ }^{\mathrm{k4} 22}$;

- coletar senhas bancárias on-line e outras informações confidenciais e enviar os dados para seu criador ${ }^{\mathrm{F} 3}$;

- acessar um computador a partir de locais remotos, roubando senhas, transações bancárias pela Internet e dados pessoais ${ }^{\mathrm{F}}$;

- criar um BHO (Objeto de Auxílio ao Navegador) o qual é executado automaticamente toda vez que o navegador web for iniciado ${ }^{\text {4s. }}$. Cabe ressaltar que os BHOs não são impedidos por firewalls pessoais porque são identificados como parte do próprio navegador. De maneira desvirtuada, os BHOs são frequentemente usados por adware e spyware visando gravar entradas de teclado e do mouse;

- localizar senhas armazenadas em um computador ${ }^{\mathrm{F} 46}$.

Características relacionadas à Antiforense Digital as quais são técnicas de remoção, ocultação e subversão de evidências com o objetivo de reduzir as consequências dos resultados de análises forense. Caso o arquivo periciado tente:

- suspender a sua própria execução até que um determinado intervalo de tempo limite tenha decorrido ${ }^{\mathrm{F} 47}$. Estratégia típica dos malwares que ficam inativos até $\mathrm{O}$ término da quarentena dos antivírus comerciais;

- desabilitar os mecanismos de defesa da vítima, inclui-se Firewall e Antivírus ${ }^{\mathrm{F} 48}$;

- desabilitar as atualizações automáticas do Windows ${ }^{\mathrm{F} 49}$;

- detectar se o próprio arquivo está sendo analisado por um depurador do Sistema Operacional ${ }^{\mathrm{F} 50}$;

- recuperar informações sobre o primeiro e o próximo processo encontrado em um snapshot do Sistema Operacional ${ }^{\text {rsi. }}$. Tal estratégia é típica de malwares que visam corromper backups e pontos de restauração do Sistema Operacional;

- ocultar um arquivo em outro. Tal estratégia é denominada, tecnicamente, de esteganografia o qual visa esconder o malware em um programa benigno no Gerenciador de Tarefas ${ }^{\mathrm{r} 2}$;

- disfarçar o seu próprio nome no Gerenciador de Tarefas ${ }^{\mathrm{F} 3}$;

- fazer uso de bibliotecas associadas ao Hackers Encyclopedia 2002 54;

- criar um cyber-ataque do tipo ZeroAcess ${ }^{\mathrm{Fs}}$ através de atualizações dos firmwares dos dispositivos de hardware (eg.: controlada do disco rígido).

Características relacionadas à criação de GUI (Graphical User Interface - Interface Gráfica do Usuário) do programa suspeito. $\mathrm{O}$ antivírus criado audita se o arquivo suspeito tenta:

- criar uma GUI em tempo de execução ${ }^{\mathrm{F} 6}$;

- usar o DirectX o qual permite que aplicativos multimídia desenhem gráficos 2D ${ }^{\mathrm{BS}}$; 
- criar módulo que contém rotinas de compactação e descompactação de bitmap usadas para o Microsoft Vídeo para Windows ${ }^{\text {s: }}$

- criar gráficos 3D relacionadas a funções utilitárias usadas pelo OpenGL ${ }^{\text {r59; }}$

- detectar formas através de visão computacional e processamento digital de imagem ${ }^{\mathrm{F}}$;

- acessar funcionalidades visando criar e gerenciar janelas de tela e controles mais básicos, como botões e barras de rolagem, receber entrada de mouse e teclado e outras funcionalidades associadas à parte de GUI do Windows. Isso inclui coisas como barras de status, barras de progresso, barras de ferramentas e guias ${ }^{\mathrm{ral}}$;

Características relacionadas à perícia ilícita da memória principal (RAM) do sistema local. $\mathrm{O}$ antivírus criado investiga se o aplicativo suspeito tenta:

- acessar informações em regiões específicas da memória principal ${ }^{\mathrm{ra}}$;

- ler dados de uma área de memória ocupada por um processo específico ${ }^{\mathrm{F}}$;

- Gravar dados em uma área de memória em um processo específico ${ }^{\mathrm{F}}$;

- Reservar, confirmar ou alterar o estado de uma região de páginas no espaço de endereço virtual de um processo ${ }^{\text {Fos }}$.

Características relacionadas ao tráfego de rede. Averígua-se se o arquivo testado tenta:

- consultar servidores DNS F66;

- enviar solicitação para um o servidor HTTP ${ }^{\mathrm{E} 67}$;

- monitorar informações dos cabeçalhos dos pacotes de dados do computador associadas a uma solicitação HTTP ${ }^{\mathrm{F} 68}$;

- enviar uma solicitação de eco IPv4 ICMP ${ }^{\text {F69; }}$

- enviar uma solicitação SNMP utilizada para monitorizar equipamentos de rede local ${ }^{\mathrm{F} 70}$;

- encerrar a conexão com a Internet ${ }^{\mathrm{F} 71}$;

- criar uma sessão FTP ou HTTP em tempo de execução ${ }^{\text {F72; }}$

- fragmentar uma URL em tempo de execução ${ }^{\mathrm{F} 73}$;

- consultar um servidor para determinar a quantidade de dados disponíveis de tráfego ${ }^{\mathrm{F} 74}$;

- identificar o estado de conexão do sistema local em relação à Internet ${ }^{\mathrm{F} 74}$;

- inicializar o uso de um aplicativo das funções do WinINet (API do Windows visando a criação e utilização de aplicação utilizando a Internet) ${ }^{\text {F75 }}$

- ler dados dos pacotes de rede feita a partir de requisições prévias do sistema local (comportamento típico de sniffers) ${ }^{\mathrm{F} 76}$;

- sobrescrever dados em um pacote de rede do sistema local ${ }^{\mathrm{F} 78}$;

- gerenciar sistemas de rede locais e remotos ${ }^{\mathrm{F} 9}$;

- criar um socket de rede no sistema local. Em uma aplicação convencional, o servidor envia dados para o(s) cliente(s). De forma inversa, nos malwares, a vítima envia os dados (imagens, dígitos) para o servidor. Logo, os malwares podem criar sockets, no sistema local, aguardando (listen) que um computador mal intencionado remoto requisite uma conexão e, portanto, possa receber as informações íntimas da vítima ${ }^{\text {во }}$ 
- Receber dados de um socket. Estratégia típica de backdoors quando a vítima passa a receber comandos (ordens) remotos ${ }^{\mathrm{rs}}$;

- Enviar dados em um socket. Estratégia típicas de spywares os quais, após a captura de informações íntimas, as enviam para um computador remoto malintencionado ${ }^{\mathrm{F} 2}$.

Características relacionadas a programas aplicativos utilitários. O antivírus criado verifica se o arquivo suspeito tenta:

- reproduzir vídeos/áudios pelo Windows Media Player ${ }^{\text {в3; }}$

- alterar ícone atalho e configurações padrões de Internet exibidos na barra de endereços da barra de ferramentas do Explorer ${ }^{\mathrm{r84}}$;

- alterar as configurações do Wordpad ${ }^{\text {вs; }}$

- alterar as configurações dos sockets, especificamente, gerenciados pelo internet Explorer ${ }^{\text {r86 }}$;

- alterar as configurações do Outlook Express e acessar a lista de contatos de e-mail da vítima ${ }^{\mathrm{r8}}$;

- acessar informações atreladas ao Microsof Office ${ }^{\text {r8s8 }}$;

- alterar as configurações do suíte da Adobe Systems ${ }^{\text {ғ9}}$;

- alterar as configurações da limpeza de disco do Sistema ғю;

- alterar as configurações de jogos eletrônicos digitais nativos além dos vinculados às empresas Tycoon e Electronic Arts;

- alterar as configurações de atualizações do Google Inc ${ }^{\text {म91; }}$

- usar o software Visual Basic ${ }^{\mathrm{r} 2}$. Tal estratégia é típica dos vírus de macro os quais são visam infectar os aplicativos que suportam linguagem de macro como os navegadores web, o Microsoft Office e o Adobe Systems.

- alterar as configurações de acesso ao Wikipédia ${ }^{\text {9:3; }}$

Através da descrição das características auditadas pelo antivírus proposto, é possível estabelecer que os malwares, em grande parte dos casos, empregam serviços lícitos nativos do sistema operacional, no entanto, de forma desvirtuada (CONRAD, et al., 2017). Os malwares, por exemplo, usam a webcam da vítima sem o seu consentimento. Conclui-se que é equivocado condenar um aplicativo por ele fazer uso de um determinado processo (eg. webcam). Em síntese, é incorreto condenar uma aplicação por conta de uma única característica, pois tal característica também pode ser usada por aplicações benignas. Então, o reconhecimento de malwares deve ocorrer através do cruzamento de informações e, consequentemente, a ponderação de todos os comportamentos auditados. O antivírus proposto pondera, estatisticamente, as características auditadas através do uso de Data Science, máquinas de aprendizado estático e inteligência artificial.

Tabela 11. Parte 1: Ferramentas, funções e bibliotecas empregadas na extração de características dos arquivos executáveis.

\begin{tabular}{l|l}
\hline Código & \multicolumn{1}{|c}{ Ferramenta } \\
\hline F01 & $\begin{array}{l}\text { Pescanner: Ferramenta de extração de características dos executáveis } \\
\text { portáveis (PE files). }\end{array}$ \\
\hline
\end{tabular}




\begin{tabular}{|c|c|}
\hline F02 & $\begin{array}{l}\text { Repertório de instruções da arquitetura de } 32 \text { bits do Windows. Disponível } \\
\text { em: https://docs.microsoft.com/en-us/windows- } \\
\text { hardware/drivers/debugger/x86-instructions. }\end{array}$ \\
\hline F03 & Bibliotecas nativas do Windows Npptools, Sprintsv. \\
\hline F04 & Função GetUserNameA da biblioteca Windows.h. \\
\hline F05 & Biblioteca Useren nativa do Windows. \\
\hline F06 & Função GetTickCount da biblioteca Windows.h. \\
\hline F07 & Biblioteca ShellExecute nativa do Windows. \\
\hline F08 & $\begin{array}{l}\text { Função GetVersionEx da biblioteca Windows.h. Biblioteca powrprof.dll } \\
\text { nativa no Windows } 10 .\end{array}$ \\
\hline F09 & Biblioteca Hook nativa do Windows. \\
\hline F10 & $\begin{array}{l}\text { Função GetStartupInfo da biblioteca Windows.h. Bibliotecas Psapi, Shell32, } \\
\text { Ole32, Oleaut32, Ws2_32, msvcrt, uxtheme, Crypt32, ntdll, Rpcrt4, Odbc32, } \\
\text { Mpr, Msvcp60, Imagehlp, netapi32, Setupapi, Iphlpapi, Dnsapi nativas do } \\
\text { Windows. }\end{array}$ \\
\hline F11 & Biblioteca Shlwapi nativa do Windows. \\
\hline F12 & Biblioteca Msimg32 nativa do Windows. \\
\hline F13 & Biblioteca comdlg32 nativa do Windows. \\
\hline F14 & Biblioteca Slmgr nativa do Windows. \\
\hline F15 & Biblioteca Rpcrt4 e MSVCP60 nativas do Windows. \\
\hline F16 & Biblioteca Powrprof nativa do Windows. \\
\hline F17 & $\begin{array}{l}\text { Bibliotecas GetThreadContext, OpenProcess, StartService, } \\
\text { TerminateProcess, WinExec e Kernel32 nativas do Windows. }\end{array}$ \\
\hline F18 & Biblioteca CertDeleteCertificateFromStore nativa do Windows. \\
\hline F19 & $\begin{array}{l}\text { Funções CopyFile, CeCopyFile, GetTempFileName, GetFileSize, } \\
\text { GetFileAttributes e GetModuleHandle da biblioteca Windows.h. }\end{array}$ \\
\hline F20 & Funções CreateFile e DeleteFile da biblioteca Windows.h. \\
\hline F21 & \begin{tabular}{lcrr} 
Bibliotecas & \multicolumn{2}{c}{ CreateProcess, } & \multicolumn{2}{c}{ ZwQueryInformationProcess, } \\
CreateToolhelp32Snapshot CreateFileMapping & CreateRemoteThread \\
CreateThread $\quad$ EnumProcesses & ExitThread & GetCommandLine \\
GetProcAddress nativas do Windows. & &
\end{tabular} \\
\hline F22 & Função CreateDirectory da biblioteca Windows.h. \\
\hline F23 & $\begin{array}{l}\text { Funções FindFirstFile, FindNextFile, FindFirstFile, FindFirstFileEx, e } \\
\text { FindFirstFileTransacted da biblioteca Windows.h. }\end{array}$ \\
\hline F24 & Função CreateService da biblioteca Windows.h. \\
\hline F25 & Bibliotecas CryptEncrypt e Crypt. \\
\hline F26 & Bibliotecas Advapi32 e Wmiprvse nativas do Windows. \\
\hline F27 & Biblioteca Msacm32 nativa do Windows. \\
\hline F28 & Biblioteca Gdi32 e Print nativa do Windows. \\
\hline F29 & Processo res.exe pertencente ao USB Storage Toolbox nativo do Windows. \\
\hline
\end{tabular}

Tabela 12. Parte 2: Ferramentas, funções e bibliotecas empregadas na extração de características dos arquivos executáveis.

\begin{tabular}{l|l}
\hline Código & \multicolumn{1}{|c}{ Ferramenta } \\
\hline F30 & Funções FindResource e FltRegisterFilter da biblioteca Windows.h. \\
\hline F31 & Funções GetDriveType e WriteFile nativas da biblioteca Windows.h. \\
\hline F32 & Função GetComputerName da biblioteca Windows.h. \\
\hline
\end{tabular}

isys: Revista Brasileira de Sistemas de Informação (isys: Brazilian Journal of Information Systems) http://seer.unirio.br/index.php/isys/ 


\begin{tabular}{|c|c|}
\hline $\mathrm{F} 33$ & Biblioteca RegCloseKey nativa do Windows. \\
\hline F34 & Biblioteca RegCreateKeyA nativa do Windows. \\
\hline $\mathrm{F} 35$ & Bibliotecas RegDeleteKey e RegDeleteValue nativas do Windows. \\
\hline F36 & Bibliotecas RegEnumKey e RegOpenKey nativas do Windows. \\
\hline F37 & Função GetUpdateRect da biblioteca Windows.h. \\
\hline F38 & Função GetUpdateRgn da biblioteca Windows.h. \\
\hline F39 & Biblioteca avicap32 nativa do Windows. \\
\hline F40 & $\begin{array}{l}\text { Biblioteca MSVBVM60 desenvolvida pela Avante } \\
\text { Technology. }\end{array}$ \\
\hline F41 & $\begin{array}{l}\text { Bibliotecas SetKeyboardState, GetKeyboardState e GetKeyState nativas do } \\
\text { Windows. Biblioteca msto } 32 \text { do cavalo de Tróia Tofger-N. }\end{array}$ \\
\hline F42 & Biblioteca mswapi vinculada ao Trojan.Iespy-A. \\
\hline F43 & Biblioteca user128 vinculada ao infostealer.pport trojan. \\
\hline F44 & $\begin{array}{l}\text { win.exe é um processo que está registrado como o cavalo de troia Agobot. } \\
\text { aim spy plugin.dll é uma biblioteca que está registrada como o Trojan } \\
\text { AIMVision pelo Digital Underground. }\end{array}$ \\
\hline $\mathrm{F} 45$ & Bibliotecas Htmledit.dl e toolband.dll. \\
\hline F46 & Ferramenta: Win32 / WEPSpy. \\
\hline F47 & Biblioteca Sleep nativa do Windows. \\
\hline F48 & Biblioteca Secur32 nativa do Windows. Bibl \\
\hline F49 & Biblioteca Urlmon.dll nativa do Windows. \\
\hline F50 & $\begin{array}{l}\text { Bibliotecas IsDebuggerPresent, } \\
\text { CheckRemoteDebuggerPresent e UnhandledExceptionFilter nativas do } \\
\text { Windows; }\end{array}$ \\
\hline F51 & Bibliotecas Process32First Process32Next. \\
\hline F52 & Processo lsasrv32.exe mal-intencionado. Biblioteca Hksrv. \\
\hline F53 & Biblioteca wininet que pode ser usado pelo trojan Troj / Zlob-AO. \\
\hline F54 & Biblioteca packet.dll. \\
\hline F55 & $\begin{array}{l}\text { Biblioteca hal.dll. O HAL (Camada de Abstração de Hardware) serve como } \\
\text { uma interface entre o hardware e o software de um sistema }\end{array}$ \\
\hline F56 & $\begin{array}{l}\text { Função GetWindowThreadProcessId da biblioteca Windows.h. Biblioteca } \\
\text { FindWindow nativa do Windows. }\end{array}$ \\
\hline F57 & Bibliotecas ddraw e Dsound. \\
\hline F58 & $\begin{array}{l}\text { Biblioteca Jpeg1x32 desenvolvida pela Eastman Software, Inc., A Kodak } \\
\text { Business para o Windows. }\end{array}$ \\
\hline F59 & Bibliotecas Gdiplus, Glu32 e Opengl32. \\
\hline F60 & $\begin{array}{l}\text { Biblioteca Msvcp71 associado ao Microsoft }{ }^{\circledR} \text { Visual Studio.NET, } \\
\text { desenvolvido pela Imagemagick. Biblioteca Quartz.dll associada ao } \\
\text { Photosuite SE }\end{array}$ \\
\hline F61 & Biblioteca comct132 nativa do Windows. \\
\hline F62 & Função LockResource nativa do Windows. \\
\hline
\end{tabular}

Tabela 13. Parte 3: Ferramentas, funções e bibliotecas empregadas na extração de características dos arquivos executáveis.

\begin{tabular}{l|l}
\hline Código & \multicolumn{1}{c}{ Ferramenta } \\
\hline F63 & Função ReadProcessMemory nativa do Windows. \\
\hline F64 & Função WriteProcessMemory nativa do Windows. \\
\hline
\end{tabular}




\begin{tabular}{l|l}
\hline F65 & Funções VirtualAlloc, VirtualAllocEx e VirtualProtect nativos do Windows. \\
\hline F66 & $\begin{array}{l}\text { Funções DNSQuery, Httpfilter, GetHostByAddr, GetHostByName e } \\
\text { GetHostName nativos do Windows. }\end{array}$ \\
\hline F67 & Funções HttpSendRequestA e Httppostda biblioteca Windows.h. \\
\hline F68 & Função HttpQueryInfoA da biblioteca Windows.h. \\
\hline F69 & Biblioteca IcmpSendEcho. \\
\hline F70 & Biblioteca snmpapi.dll. \\
\hline F71 & Função InternetCloseHandle da biblioteca Windows.h. \\
\hline F72 & Funções InternetConnect FtpGetFile FtpOpenFile da biblioteca Windows.h. \\
\hline F73 & Biblioteca InternetCrackUrlA. \\
\hline F74 & Biblioteca InternetQueryDataAvailable. \\
\hline F75 & Biblioteca InternetGetConnectedState. \\
\hline F76 & Biblioteca InternetOpenA. \\
\hline F77 & Biblioteca InternetReadFile. \\
\hline F78 & Biblioteca InternetWriteFile. \\
\hline F79 & Biblioteca WMIPRVI: Windows Management Instrumentation. \\
\hline F80 & $\begin{array}{l}\text { Funções listen, socket, WSASend, WSASocket e WSAStartup. Bibliotecas } \\
\text { Wsock32 e Ws2help. }\end{array}$ \\
\hline F81 & Função recv nativa do Windows.h. \\
\hline F82 & Função send nativa do Windows.h. \\
\hline F83 & Biblioteca Wmvcore nativa do Windows. \\
\hline F84 & Bibliotecas Ur,l Comdlg32, IEHelper e Oleacc nativas do Windows. \\
\hline F85 & Biblioteca Oledlg nativa do Windows. \\
\hline F86 & Bibliotecas Mswsock e Ieloader nativas do Windows. \\
\hline F87 & Bibliotecas Atl, WAB32 e wab32. \\
\hline F88 & Bibliotecas MSVCIRT e MSO9. \\
\hline F89 & Processo bridge.exe e biblioteca Plugin.dll. \\
\hline F90 & Processo cleanmgr.exe nativo do Windows. \\
\hline F91 & Biblioteca goopdate. \\
\hline F93 & Biblioteca msvbvm50. \\
Cygcrypt-0.dll é um tipo de arquivo DLL associado ao Wiki Web \\
\hline
\end{tabular}

\subsection{Classificadores}

Quanto ao reconhecimento de padrão de malwares, uma tarefa essencial diz respeito à atribuição de uma classe (rótulo) a cada arquivo investigado a partir de suas características. Então, com base em um conjunto de arquivos, chamado de conjunto de treinamento, é possível formular uma hipótese sobre as distintas classes atreladas ao antivírus inteligente proposto. Logo, cabe ao classificador estimar a classe de um arquivo inédito através da comparação entre as características do seu comportamento auditado e àquelas captadas durante a sua etapa de treinamento.

O objetivo do classificador é obter uma função de separação entre as classes do antivírus criado (malware, benigno). Dessa forma, ao ser apresentado a um aplicativo inédito, a função é aplicada e, então, atribui-se uma classe na qual esse aplicativo suspostamente pertence. Matematicamente, $c=f(x)$, onde $x=x_{1}, x_{2}, \ldots, x_{t}$ é um vetor 
características extraídas do arquivo investigado, $t$ corresponde às 630 características auditadas, $c$ é a classe (rótulo), por fim, $f$ é a função de mapeamento do classificador.

O trabalho proposto emprega redes neurais artificiais como classificadores. Especificamente, são empregadas as redes neurais do tipo MLP (Multilayer Perceptron Perceptron com Múltiplas Camadas) dotadas de retropropagação. Uma rede MLP é uma generalização da rede Perceptron simples pela adição de, ao menos, uma camada intermediária (HAGAN, et al., 1996). Convencionalmente, as redes MLP apresentam 3 (três) camadas: uma camada de entrada, ao menos uma camada intermediária e uma camada de saída. Na camada de entrada, cada neurônio de entrada representa uma variável considerada como entrada para o problema. No antivírus inteligente criado, há 630 neurónios e dizem respeito a comportamentos maliciosos descritos na subseção 3.2. Na camada escondida, há o encargo pela não linearidade da rede neural. Por fim, na camada de saída, há o reconhecimento do padrão quanto à resposta da rede e representa a variável desejada. No antivírus inteligente criado, a camada de saída possui dois neurônios correspondentes aos aplicativos benignos e malwares.

As redes MLP, baseadas em retropropagação, utilizam basicamente dois passos. No primeiro passo, há a propagação de dados (impulso sináptico) da camada de entrada para a camada de saída. Nesse passo, há o cálculo do sinal de saída e o erro (HAGAN, et al., 1996). O erro é a diferença entre o sinal de saída obtida e a saída desejada. Após isso, no segundo passo, há a propagação de dados partindo da camada de saída em direção à camada de entrada. Esse passo é conhecido com retropropagação e visa ajustar os pesos referentes às ligações sinápticas entre os neurônios. Cabe ressaltar que os pesos são determinados, inicialmente, de forma aleatória.

Com o objetivo de otimizar a precisão do antivírus criado, são averiguadas onze diferentes funções de aprendizado $f$ baseadas em classificadores MLP dotados de retropropagação. A seguir, são detalhadas todas as onze diferentes formas de implementações das redes MLPs empregadas pelo trabalho proposto.

1. Lotes de peso e limite bias: emprega atualizações em lotes, que são atualizados ao final de cada época (HAGAN, et al., 1996).

2. Gradiente Powell-Beale: emprega o método de retropropagação do gradiente conjugado baseado na técnica Powell-Beale (POWELL, 1977).

3. Retropropagação Fletcher-Powell: utiliza o método de retropropagação do gradiente conjugado baseado na técnica Fletcher-Reeves (NOTAY, 2000).

4. Retropropagação Polak-Ribiere: aplica o método de retropropagação do gradiente conjugado inspirado na técnica Polak-Ribiere (SCALES, 1985).

5. Retropropagação decrescente: emprega o método de retropropagação baseado em um gradiente descendente (HAGAN, et al., 1996).

6. Retropropagação com momentos: emprego o método de retropropagação baseado em um gradiente descendente com momentos. Esse classificador permite que a rede neural não apenas se baseie em informações locais mas também tenha a capacidade de ignorar ruídos do gradiente. $\mathrm{O}$ objetivo é que a rede neural não se atenha a um mínimo local (HAGAN, et al., 1996). 
7. Retropropagação com taxa adaptativa: implementa uma taxa de aprendizagem adaptativa a qual se guia baseada nas informações do gradiente. Uma taxa de aprendizagem fixa pode apresentar problemas durante o treinamento. Um taxa de aprendizagem fixa muito alta, o classificador pode oscilar e se tornar instável. Por outro lado, caso a taxa de aprendizagem fixa for muito baixa, o tempo de treinamento pode se tornar bastante elevado (HAGAN, et al., 1996).

8. Retropropagação combinada: é a junção da retropropagação com taxa adaptativa com a retropropagação baseada em momentos. Isso quer dizer, essa rede neural não se baseia apenas em informações locais como também possui um taxa de aprendizagem adaptativa (HAGAN, et al., 1996).

9. Retropropagação secante em um passo: implementa a retropropagação do gradiente conjugado baseado na técnica da secante em um único passo (BATTITI, 1992).

10. Retropropagação resiliente (Rprop): emprega o método de retropropagação do gradiente conjugado baseado no algoritmo Rprop (RIEDMILLER, et al., 1993).

11. Retropropagação gradiente escalonado: emprego o método de retropropagação do gradiente conjugado escalonado (MOLLER, 1993).

Nas redes neurais MLP, condições iniciais convenientes podem fazer com que a rede convirja em poucas épocas. Por outro lado, condições iniciais inadequadas podem demandar uma grande quantidade de épocas até a rede adquirir capacidade de generalização e, portanto o treinamento ser encerrado. Então, um cuidado metodológico, adotado pelo trabalho proposto, é averiguar a influência das condições iniciais nos resultados alcançados dos classificadores. Logo, são utilizados 30 (trinta) diferentes pesos iniciais aleatórios referentes às ligações sinápticas entre os neurônios. As variações são aleatórias com a semente do gerador aleatório entre 1 e 30 de maneira incremental.

A quantidade de pesos iniciais foi estabelecida como 30 (trinta) sementes de gerador aleatório. A motivação é que 30 (trinta) iterações constituem uma quantidade estatisticamente significativa de modo a se determinar o grau de dispersão das amostras. Então, para cada rede neural (função de aprendizado), são avaliadas 30 (trinta) amostras assim como ocorrem em diversas áreas como, por exemplo, em Engenharia Biomédica (LIMA, et al., 2016). Na Tabela dos resultados, no capítulo seguinte, a dispersão é representada através do desvio padrão das 30 (trinta) iterações de cada função de aprendizado avaliada. Então, quanto menor o desvio padrão implica que a rede neural avaliada não sofre mudanças abruptas em função dos pesos iniciais aleatórios referentes às ligações sinápticas entre os neurônios. De modo oposto, quanto maior for o desvio padrão significa que a rede neural sobre brusca alteração, em sua precisão, em função dos pesos iniciais das ligações sinápticas.

O trabalho proposto faz uso de três arquiteturas para cada MLP. A primeira arquitetura utiliza uma única camada escondida, com 100 neurônios. A segunda arquitetura utiliza duas camadas escondidas, com 100 neurônios cada. Os resultados da segunda abordagem são simbolizados com um asterisco. Por fim, a terceira arquitetura emprega uma camada escondida, no entanto, são utilizados 500 neurônios. A terceira abordagem é simbolizada através de dois asteriscos na tabela que exibirá os resultados alcançados. A hipótese é verificar se o aumento da quantidade de neurônios e, consequentemente, o aumento de cálculos numéricos da rede neural é capaz de prover 
melhores resultados. Para cada função de aprendizado, há 90 (3 arquiteturas * 30 pesos iniciais) iterações. São exploradas 3 (três) tipos de arquiteturas e 30 (trinta) diferentes pesos iniciais, referentes às ligações sinápticas entre os neurônios, para cada arquitetura.

Quanto aos dados de entrada, 70\% dos dados são destinados ao treinamento, 15\% são reservados à validação cruzada, por fim $15 \%$ são utilizados na fase de testes. $O$ número máximo de épocas, para treinamento, é 1000 (um mil). A validação cruzada ocorre após cada época com o objetivo da rede neural não perder a sua capacidade de generalização. Há uma tolerância de 5 (cinco) falhas para a validação cruzada. O tempo de treinamento das MLPs engloba, obviamente, o tempo gasto no treinamento juntamente com o tempo destinado à validação cruzada.

\section{Resultados}

A Tabela 14 exibe a classificação dos resultados usando as redes MLP baseadas em retropropagação. Os melhores casos são grifados em negrito. O primeiro e segundo critérios são a média aritmética e o desvio padrão, respectivamente. São utilizadas onze diferentes funções de aprendizado. Para cada função, são estudadas três tipos distintos de arquitetura, descritas na seção 3.2.1. Quanto ao tempo de treinamento, os classificadores apresentam tempos elevados assim como os desvios padrões. Esse fato indica que as MLPs sofrem variações abruptas, em relação ao tempo de treinamento, a depender dos pesos iniciais referentes às ligações sinápticas entre os neurônios. Conforme estabelecido anteriormente, "“, “*”, “**” correspondem às arquiteturas com 1 (uma) camada escondida com 100 neurônios, 2 (duas) camadas escondidas cada qual com 100 neurônios e 1 (uma) camada escondida com 500 neurônios, respectivamente.

No tocante à precisão, a mínima taxa de acerto foi de 48,87\% para a rede que emprega o Rprop (Retroprogação Resiliente). Essa abordagem possui uma arquitetura com duas camadas escondidas com 100 neurônios cada uma. Enquanto, a máxima precisão foi de $98,13 \%$ para a rede inspirada em retropropagação de Gradiente Escalonado. A arquitetura, dessa rede, contém uma camada escondida com 100 neurônios. Note também que o desvio padrão é de 0,68 , um valor relativamente baixo. Isso corrobora o excelente desempenho da função de aprendizado do Gradiente Escalonado, independente das condições iniciais.

Quanto ao tempo de treinamento, os classificadores baseados em retropropagação apresentam desvios padrões altos. Isso indica que as redes MLPs sofrem variações abruptas, em relação a tempo de aprendizado, a depender do conjunto de pesos iniciais. Observa-se que, em regra geral, ao expandir a quantidade de neurônios na camada escondida, também cresce o tempo de treinamento devido a um maior volume de cálculos da rede neural.

Quanto às arquiteturas das redes, aumentar a quantidade de camadas escondidas ou aumentar a sua quantidade de neurônios, não implica em um melhor desempenho de maneira sistemática. Em várias situações, aumentar a quantidade de neurônios na camada escondida provocou uma degradação no desempenho acompanhada de um maior tempo destinado ao treinamento devido ao grande volume de cálculos. Logo, a exploração de diferentes tipos de arquiteturas de redes neurais foi uma decisão salutar visto que não houve uma arquitetura ótima independente da variação da função de aprendizado. 
Tabela 14. Resultados das redes baseadas em retropropagação para classificação em duas classes de executáveis: benignos e malwares.

\begin{tabular}{|c|c|c|c|c|}
\hline & $\begin{array}{c}\text { Acerto treino } \\
(\%)\end{array}$ & $\begin{array}{c}\text { Acerto } \\
\text { teste }(\%)\end{array}$ & $\begin{array}{c}\text { Tempo treino } \\
\text { (seg.) }\end{array}$ & $\begin{array}{c}\text { Tempo } \\
\text { teste (seg.) }\end{array}$ \\
\hline Lotes de peso e limite bias & $54,12 \pm 14,30$ & $54,11 \pm 14,11$ & $27,90 \pm 79,20$ & $0,06 \pm 0,04$ \\
\hline Lotes de peso e limite bias* & $56,27 \pm 15,38$ & $56,21 \pm 15,21$ & $26,60 \pm 84,70$ & $0,06 \pm 0,04$ \\
\hline Lotes de peso e limite bias** & $53,81 \pm 11,43$ & $53,79 \pm 11,35$ & $40,16 \pm 199,11$ & $0,11 \pm 0,4$ \\
\hline Gradiente Powell-Beale & $96,95 \pm 4,19$ & $96,31 \pm 4,28$ & $41,44 \pm 24,33$ & $0,05 \pm 0,03$ \\
\hline Gradiente Powell-Beale* & $96,75 \pm 3,60$ & $96,21 \pm 3,66$ & $41,65 \pm 23,43$ & $0,07 \pm 0,05$ \\
\hline Gradiente Powell-Beale** & $96,70 \pm 4,09$ & $96,25 \pm 3,92$ & $76,19 \pm 40,99$ & $0,10 \pm 0,04$ \\
\hline Retropropagação Fletcher-Powell & $97,41 \pm 4,64$ & $96,55 \pm 4,60$ & $61,79 \pm 32,72$ & $0,06 \pm 0,03$ \\
\hline Retropropagação Fletcher-Powell* & $98,32 \pm 2,95$ & $97,60 \pm 2,99$ & $85,20 \pm 43,79$ & $0,05 \pm 0,04$ \\
\hline Retropropagação Fletcher-Powell** & $98,33 \pm 3,40$ & $97,54 \pm 3,42$ & $165,77 \pm 75,37$ & $0,08 \pm 0,04$ \\
\hline Retropropagação Polak-Ribiere & $96,96 \pm 34,6$ & $96,39 \pm 3,47$ & $49,81 \pm 25,73$ & $0,05 \pm 0,03$ \\
\hline Retropropagação Polak-Ribiere* & $97,15 \pm 3,00$ & $96,69 \pm 3,01$ & $62,92 \pm 30,45$ & $0,06 \pm 0,05$ \\
\hline Retropropagação Polak-Ribiere** & $96,96 \pm 2,11$ & $96,54 \pm 1,96$ & $112,39 \pm 65,00$ & $0,10 \pm 0,06$ \\
\hline Retropropagação decrescente & $83,11 \pm 14,64$ & $82,94 \pm 14,46$ & $43,09 \pm 55,64$ & $0,06 \pm 0,04$ \\
\hline Retropropagação decrescente* & $77,40 \pm 16,13$ & $76,63 \pm 16,23$ & $61,97 \pm 89,61$ & $0,06 \pm 0,04$ \\
\hline Retropropagação decrescente $* *$ & $52,86 \pm 13,28$ & $52,77 \pm 12,94$ & $19,23 \pm 82,56$ & $0,10 \pm 0,04$ \\
\hline Retropropagação com momentos & $55,29 \pm 14,34$ & $55,17 \pm 14,22$ & $13,98 \pm 38,29$ & $0,05 \pm 0,05$ \\
\hline Retropropagação com momentos* & $51,63 \pm 10,90$ & $51,67 \pm 10,87$ & $8,82 \pm 37,48$ & $0,05 \pm 0,05$ \\
\hline Retropropagação com momentos** & $50,16 \pm 4,72$ & $50,28 \pm 4,80$ & $1,98 \pm 0,40$ & $0,09 \pm 0,04$ \\
\hline Retropropagação com taxa adaptativa & $81,18 \pm 07,42$ & $80,68 \pm 7,29$ & $6,74 \pm 2,54$ & $0,05 \pm 0,04$ \\
\hline Retropropagação com taxa adaptativa* & $85,63 \pm 3,59$ & $84,94 \pm 3,24$ & $12,44 \pm 2,66$ & $0,07 \pm 0,04$ \\
\hline Retropropagação com taxa adaptativa** & $78,17 \pm 11,40$ & $77,96 \pm 11,29$ & $35,53 \pm 21,23$ & $0,09 \pm 0,04$ \\
\hline Retropropagação combinado & $88,92 \pm 8,28$ & $87,96 \pm 8,11$ & $14.00 \pm 3,56$ & $0,06 \pm 0,04$ \\
\hline Retropropagação combinado* & $89,09 \pm 6,27$ & $88,11 \pm 6,18$ & $19,46 \pm 7,12$ & $0,06 \pm 0,04$ \\
\hline Retropropagação combinado** & $86,37 \pm 12,56$ & $85,69 \pm 12,16$ & $51,85 \pm 20,89$ & $0,09 \pm 0,03$ \\
\hline Retropropagação secante em um passo & $98,60 \pm 1,17$ & $98,00 \pm 0,92$ & $70,19 \pm 25,57$ & $0,06 \pm 0,04$ \\
\hline Retropropagação secante em um passo* & $98,36 \pm 1,25$ & $97,76 \pm 0,99$ & $92,30 \pm 33,48$ & $0,05 \pm 0,04$ \\
\hline Retropropagação secante em um passo** & $97,47 \pm 1,50$ & $97,04 \pm 1,27$ & $168,50 \pm 67,58$ & $0,10 \pm 0,04$ \\
\hline Retropropagação resiliente (Rprop) & $50,67 \pm 4,16$ & $50,70 \pm 3,92$ & $0,68 \pm 0,11$ & $0,07 \pm 0,04$ \\
\hline Retropropagação resiliente (Rprop)* & $48,82 \pm 7,03$ & $48,87 \pm 6,83$ & $1,01 \pm 0,17$ & $0,06 \pm 0,04$ \\
\hline Retropropagação resiliente (Rprop)** & $51,82 \pm 4,61$ & $51,57 \pm 4,38$ & $1,47 \pm 0,16$ & $0,10 \pm 0,04$ \\
\hline Retropropagação gradiente escalonado & $98,82 \pm 0,95$ & $98,13 \pm 0,68$ & $30,92 \pm 6,69$ & $0,06 \pm 0,04$ \\
\hline Retropropagação gradiente escalonado* & $98,57 \pm 1,06$ & $98,05 \pm 0,81$ & $40,82 \pm 11,40$ & $0,06 \pm 0,04$ \\
\hline Retropropagação gradiente escalonado** & $97,59 \pm 1,51$ & $97,17 \pm 1,24$ & $65,00 \pm 26,83$ & $0,09 \pm 0,05$ \\
\hline
\end{tabular}

Então, a inteligência artificial se torna uma boa alternativa para as fabricantes dos antivírus comerciais. A inteligência artificial, durante sua etapa de aprendizado, é capaz de analisar milhares de malwares e extrair características deles. Especificamente, no antivírus inteligente proposto, são extraídas 630 características referentes ao algoritmo do arquivo suspeito. Então, após o aprendizado, caso um novo arquivo, ainda não catalogado, fosse investigado, haveria a possibilidade dele ser corretamente classificado, como malware. O correto reconhecimento de padrão se dá através da comparação entre as características do seu algoritmo e as aquelas captadas durante o processo de aprendizado da inteligência artificial.

Um antivírus, ao empregar inteligência artificial, consegue automatizar a análise de centenas de características de arquivos suspeitos em larga escala e em tempo real. Logo, não seria mais necessário aguardar que algum cliente fosse infectado e, em 
sequência denunciasse um comportamento anômalo de seu dispositivo, para, então, tomar-se providências quanto à detecção de um novo malware. Cabe salientar que as malfeitorias dos malwares podem ser irreversíveis e irrecuperáveis. Logo, um antivírus deve detectar as pragas virtuais de forma preventiva ao invés de reativa. A inteligência artificial possibilita a detecção preventiva da praga virtual antes mesmo dela ser executada pela cliente. $\mathrm{O}$ antivírus inteligente criado alcança um desempenho médio de 98,13\% na distinção entre executáveis benignos e malwares, acompanhado de um tempo de resposta médio de apenas 0,06 segundos.

\section{Conclusão}

Sistematicamente, ao longo da década, a produção de malwares é crescente (CERT.BR, 2016). Então, é importante que os antivírus provenham serviços robustos, em larga escala e em tempo real, com o objetivo de coletar amostras maliciosas recém-criadas visando proteger seus respectivos clientes. Conclui-se que a escolha de um antivírus adequado exerce papel significante na proteção contra invasões cibernéticas, visto que a detecção de malwares variou entre $0 \%$ e $99,11 \%$, a depender do antivírus comercial investigado, conforme detalhado no capítulo 2. O trabalho proposto investigou 86 antivírus comerciais. Em média, eles foram capazes de detectar $54,84 \%$ das pragas virtuais avaliadas. Com aspecto desfavorável, os antivírus, em média, atestaram falsos negativos e foram omissos em $14,34 \%$ e $30,82 \%$ dos casos, respectivamente.

O trabalho proposto empregou o VirusTotal como plataforma para submissão automatizada dos malwares aos antivírus comerciais. No VirusTotal, não há a possibilidade de escolha da versão gratuita dos antivírus. Logo, como limitação do estudo proposto, não foi possível averiguar a diferença dos serviços providos pelas versões pagas e as gratuitas de um mesmo antivírus. A suposição é que as versões gratuitas disponibilizam serviços significativamente inferiores às versões pagas.

Cerca de $17 \%$ dos antivírus avaliados não foram capazes de diagnosticar qualquer uma das amostras maliciosas. Nota-se, a limitação dos antivírus comerciais quanto à robustez de serviços em larga escala mesmo em suas versões completas (pagas). Cabe salientar que os malwares, pertencentes à base criada REWEMA, são de domínio público, largamente empregados em atividades maliciosas e com as suas atuações amplamente divulgadas em fóruns, blogs e demais conteúdos online. Mesmo assim, 17\% dos antivírus comerciais avaliados não tinham qualquer conhecimento sobre as suas existências.

O trabalho proposto desenvolve um antivírus, dotado de inteligência artificial, visando a classificação quanto a executáveis de arquiteturas de 32 bits entre benignos e malwares. A extração de características passa pelo processo de disassembling visando reverter o arquivo binário em seu código fonte. A classificação é baseada em redes neurais baseadas em retropropagação. As condições iniciais e arquiteturas são alternadas com o objetivo de maximizar a precisão dos classificadores.

Quanto à classificação, a função de aprendizado dotada de retropropagação de Gradiente Escalonado apresenta a maior precisão dentre todos os classificadores averiguados, com uma precisão média de 98,13\%. Essa abordagem possui uma arquitetura com única camada escondida de 100 neurônios. O classificador de mais baixa precisão foi de $48,87 \%$, referente à função de aprendizado Rprop (Retroprogação Resiliente). Esse pior cenário ocorre quando o Rprop é formado por uma arquitetura com duas camadas escondidas com 100 neurônios. Conclui-se que a melhor abordagem é 
quase 100,00\% superior ao pior cenário obtido. Logo, a escolha de uma adequada função de aprendizado, composta por uma correta arquitetura, é essencial para maximizar a precisão quanto à identificação de malwares.

A grande contribuição do trabalho proposto, em relação ao estado-da-arte, é suprir as limitações e imprecisões dos modelos empíricos e heurísticos comumente empregados na detecção de pragas virtuais (PRADO, et al., 2016). Então, ao invés da intuição do analista, o peso ponderado referente a um comportamento suspeito é determinado através de redes neurais artificiais. $O$ trabalho proposto constata que a inteligência artificial pode contribuir bastante para o avanço da segurança da informação em dispositivos digitais. Espera-se que o antivírus inteligente proposto atue de forma preventiva e impeça que os malwares burlem os mecanismos de defesa da vítima, inclui-se firewall e plugins de segurança.

Como limitação, o antivírus inteligente criado somente é capaz de detectar malwares, especificamente, para arquitetura de 32 bits do Sistema Operacional Windows. A explicação é que cada Sistema Operacional tem seu próprio repertório de instruções, bibliotecas e APIs. Então, por exemplo, os repertórios de instruções do Windows e do Linux são diferentes. Logo, a extração de características para aplicativos Windows, conforme detalhada na seção 3.2, não serve para Linux ou para Android. Conclui-se que o antivírus inteligente proposto não teria validade mediante malwares dos sistemas Linux e Android.

Então, a meta futura diz respeito à criação de novos antivírus, baseados em inteligência artificial, no sentido de suas aplicações a outros tipos de sistemas operacionais além do Windows. A intenção é estender a metodologia proposta a arquivos executáveis do sistema Android visto que smartphones e tablets móveis estão gradativamente se tornando indispensáveis na vida diária. O Android, por ser um sistema operacional relativamente recente, possibilita que incontáveis malwares se escondam em uma grande quantidade de aplicações legítimas, o que ameaça de forma grave a segurança do sistema e afeta diretamente o usuário.

\section{Referências}

ADKINS, F.; JONES, L.; MARTIN, C.; UPCHURCH, J. Heuristic Malware Detection Via Basic Block Comparison. 8th International Conference on In Malicious and Unwanted Software: "The Americas"(MALWARE), páginas 11-18, 2013.

ALAZAB, M. Profiling and Classifying the Behavior of Malicious Codes Journal of Systems and Software. Volume 100, Páginas 91-102, 2015.

BATTITI, R. First and second order methods for learning: Between steepest descent and Newton's method. Neural Computation, volume. 4, número 2, pág. 141-166 , 1992.

CERT.BR. Incidentes Reportados ao CERT.br. Disponível em: http://www.cert.br/stats/incidentes/2014-jan-dec/analise.html. Acesso em outubro de 2016, 2016.

CONRAD, E.; MISENAR, S.; AND FELDMAN, J. Eleventh Hour CISSP (Certified Information Systems Security Professional), Elsevier, 2017.

FAN, Y.; YE, Y.; CHEN, L. Malicious Sequential Pattern Mining for Automatic Malware Detection. Expert Systems with Applications. Volume 52, número 15, páginas 16-25, 2016. 
FILHO, D. S. F.; AFONSO, V. M.; MARTINS, V. F.; GRÉGIO, A. R. A.; GEUS, P. L.; JINO, M.; SANTOS, R. D. C. Técnicas para Análise Dinâmica de Malware. Simpósio Brasileiro de Segurança da Informação e de Sistemas Computacionais. ISBN: 978-857669-259-1, 2011.

HAGAN, M. T.; H.B., D.; M.H., B. Neural Network Design. Boston, MA: PWS Publishing, 1996.

HENKE, M.; SANTOS, C.; NUNAN, E.; FEITOSA, E.; SANTOS, E.; SOUTO, E. Aprendizagem de Máquina para Segurança de Computadores: Métodos e Aplicações. Simpósio Brasileiro de Segurança da Informação e de Sistemas Computacionais. ISBN: 978-85-7669-259-1, 2011.

INTEL. McAfee Labs: Relatório sobre ameaças. Disponível em: https://secure.mcafee.com/br/resources/reports/rp-quarterly-threats-mar2017.pdf?cid=BHP-075, 2017.

KHODAMORADI, P.; FAZLALI, M.; MARDUKHI, F.; AND NOSRATI, M. Heuristic Metamorphic Malware Detection based on Statistics of Assembly Instructions using Classification Algorithms. 18th CSI International Symposium on Computer Architecture and Digital Systems (CADS), páginas 1-6, 2015.

LIMA, S. M. L.; SILVA-FILHO, A. G.; SANTOS, W. P. Detection and classification of masses in mammographic images in a multi-kernel approach Computer Methods and Programs in Biomedicine. Volume 134, página 11-29, 2016.

MICROSOFT. Microsoft Computing Safety Index WorldWide Report, Disponível em: https://news.microsoft.com/pt-br/microsoft-lanca-o-indice-de-cidadania-digital-eincentiva-as-pessoas-a-ter-mais-empatia-online/. Acesso em junho de 2018 , 2014.

MOLLER, F. M. A scaled conjugate gradient algorithm for fast supervised learning Neural Networks. Volume 6, pág. 525-533, 1993.

NOTAY, Y. Flexible Conjugate Gradients. SIAM Journal on Scientific Computing 22 (4): 1444. doi:10.1137/S1064827599362314, 2000.

POWELL, M. J. D. Restart procedures for the conjugate gradient method. Mathematical Programming. Volume 12, pág. 241-254, 1977.

PRADO, N.; PENTEADO, U.; GRÉGIO, A. Metodologia de Detecção de Malware por Heurísticas Comportamentais. Simpósio Brasileiro em Segurança da Informação e de Sistemas. ISSN: 2176-0063, 2016.

REWEMA. REWEMA (Retrieval of 32-bit Windows Architecture Executables Applied to Malware Analysis - Redistribuição de Executáveis do Windows em Arquiteturas de 32-bits Aplicados a Análise de Malware) Disponível em: https://github.com/rewema/rewema. Acesso em janeiro de 2019.

RIEDMILLER, M.; BRAUN, H. A direct adaptive method for faster backpropagation learning: the RPROP algorithm. Proceedings of the IEEE International Conference on Neural Networks (ICNN). San Francisco, pág. 586-591, 1993.

SANS. SANS Institute InfoSec Reading Room. Out with The Old, In with The New: Replacing Traditional Antivirus, Disponível em: https://www.sans.org/reading- 
room/whitepapers/analyst/old-new-replacing-traditional-antivirus-37377. Acesso em junho de 2017, 2018.

SCALES, L. E. Introduction to Non-Linear Optimization. New York, Springer-Verlag, 1985. 